TRANSACTIONS OF THE

AMERICAN MATHEMATICAL SOCIETY

Volume 359, Number 2, February 2007, Pages 567-589

S 0002-9947(06)04030-X

Article electronically published on June 13, 2006

\title{
CHARACTERIZATIONS OF FUNCTION SPACES ON THE SPHERE USING FRAMES
}

\author{
FENG DAI
}

\begin{abstract}
In this paper we introduce a polynomial frame on the unit sphere $\mathbb{S}^{d-1}$ of $\mathbb{R}^{d}$, for which every distribution has a wavelet-type decomposition. More importantly, we prove that many function spaces on the sphere $\mathbb{S}^{d-1}$, such as $L^{p}, H^{p}$ and Besov spaces, can be characterized in terms of the coefficients in the wavelet decompositions, as in the usual Euclidean case $\mathbb{R}^{d}$. We also study a related nonlinear $m$-term approximation problem on $\mathbb{S}^{d-1}$. In particular, we prove both a Jackson-type inequality and a Bernstein-type inequality associated to wavelet decompositions, which extend the corresponding results obtained by R. A. DeVore, B. Jawerth and V. Popov ("Compression of wavelet decompositions", Amer. J. Math. 114 (1992), no. 4, 737-785).
\end{abstract}

\section{INTRODUCTION AND SUMMARY OF MAIN RESULTS}

1.1. Notations and basic facts. We start with some necessary notations. Given an integer $d \geq 3$, we denote by $\mathbb{S}^{d-1}$ the unit sphere of the $d$-dimensional Euclidean space $\mathbb{R}^{d}$ and $d \sigma(x)$ the usual Lebesgue measure on $\mathbb{S}^{d-1}$ normalized by $\int_{\mathbb{S}^{d-1}} d \sigma(x)=1$. For $0<p \leq \infty$, we let $L^{p} \equiv L^{p}\left(\mathbb{S}^{d-1}\right)$ denote the usual Lebesgue space on $\mathbb{S}^{d-1}$ endowed with the quasi-norm $\|\cdot\|_{p}$ and $H^{p} \equiv H^{p}\left(\mathbb{S}^{d-1}\right)$ the usual Hardy space on $\mathbb{S}^{d-1}$ endowed with the quasi-norm $\|\cdot\|_{H^{p}}$ (see Section 4 for a precise definition of $\left.H^{p}\left(\mathbb{S}^{d-1}\right)\right)$. Given a measurable subset $E$ of $\mathbb{S}^{d-1}$, we denote by $|E|$ its Lebesgue measure and $\chi_{E}$ its characteristic function. For $x, y \in \mathbb{S}^{d-1}$, we shall use the notation $d(x, y)$ to denote the geodesic distance $\arccos x \cdot y$ between $x$ and $y$. Moreover, we denote by $B(x, r):=\left\{y \in \mathbb{S}^{d-1}: d(x, y) \leq r\right\}$ the spherical cap with center $x \in \mathbb{S}^{d-1}$ and radius $r \in(0, \pi)$, and $M(f)$ the usual Hardy-Littlewood maximal function on $\mathbb{S}^{d-1}$ :

$$
M(f)(x):=\sup _{0<r \leq \pi} \frac{1}{|B(x, r)|} \int_{B(x, r)}|f(y)| d \sigma(y), \quad x \in \mathbb{S}^{d-1}, \quad f \in L\left(\mathbb{S}^{d-1}\right) .
$$

We let $\mathcal{S} \equiv \mathcal{S}\left(\mathbb{S}^{d-1}\right)$ denote the set of indefinitely differentiable functions on $\mathbb{S}^{d-1}$ endowed with the usual test function topology and let $\mathcal{S}^{\prime} \equiv \mathcal{S}^{\prime}\left(\mathbb{S}^{d-1}\right)$ be the dual of $\mathcal{S}$. $\mathcal{S}$ is called the space of test functions and $\mathcal{S}^{\prime}$ the space of distributions. We denote by $\langle f, \varphi\rangle$ the pairing between a distribution $f \in \mathcal{S}^{\prime}$ and a test function $\varphi \in \mathcal{S}$. Throughout the paper, the notation $\# \Lambda$ denotes the cardinality of a given finite set $\Lambda$, the letter $C$ denotes a general positive constant depending only on the

Received by the editors October 20, 2004.

2000 Mathematics Subject Classification. Primary 41A63, 42C15; Secondary 41A17, 46E35.

Key words and phrases. Spherical frames, wavelet decomposition, spherical harmonics, Besov spaces, nonlinear approximation.

The author was supported in part by the NSERC Canada under grant G121211001.

(C)2006 American Mathematical Society Reverts to public domain 28 years from publication 
parameters indicated as subscripts, and the notation $A \asymp B$ means that there are two inessential positive constants $C_{1}, C_{2}$ such that $C_{1} A \leq B \leq C_{2} A$.

For each nonnegative integer $k$, let $\mathcal{H}_{k}$ denote the space of spherical harmonics of degree $k$ on $\mathbb{S}^{d-1}$ and $Y_{k}$ the orthogonal projection of $L^{2}\left(\mathbb{S}^{d-1}\right)$ onto $\mathcal{H}_{k}$. As is well known, for $f \in L^{2}\left(\mathbb{S}^{d-1}\right)$,

$$
Y_{k}(f)(x)=\int_{\mathbb{S}^{d-1}} f(y) P_{k}(x \cdot y) d \sigma(y), \quad x \in \mathbb{S}^{d-1},
$$

where for $x=\left(x_{1}, x_{2}, \ldots, x_{d}\right), y=\left(y_{1}, y_{2}, \ldots, y_{d}\right) \in \mathbb{R}^{d}, x \cdot y=x_{1} y_{1}+x_{2} y_{2}+\ldots+$ $x_{d} y_{d}$,

$$
P_{k}(t):=\frac{2 k+d-2}{d-2} P_{k}^{\frac{d-2}{2}}(t), \quad t \in[-1,1],
$$

and $P_{k}^{\frac{d-2}{2}}(t)$ denotes the usual ultraspherical polynomial of order $\frac{d-2}{2}$ normalized by $P_{k}^{\frac{d-2}{2}}(1)=\frac{\Gamma(k+d-2)}{\Gamma(d-2) \Gamma(k+1)}$. (For a precise definition of ultraspherical polynomials, we refer to [Sz, p. 81].) Evidently, the formula (1.1) allows us to extend the definition of $Y_{k}(f)$ to contain all distributions $f \in \mathcal{S}^{\prime}\left(\mathbb{S}^{d-1}\right)$. For an integer $n \geq 0$ we denote by $\Pi_{n}$ the space of all spherical polynomials of degree at most $n$ on $\mathbb{S}^{\bar{d}-1}$ (i.e., polynomials in $d$-variables of total degree at most $n$ restricted to $\mathbb{S}^{d-1}$ ). It is well known that $\Pi_{n}=\bigoplus_{k=0}^{n} \mathcal{H}_{k}$ and $\operatorname{dim} \Pi_{n}=\sum_{k=0}^{n} \operatorname{dim} \mathcal{H}_{k} \asymp n^{d-1}$. For more information on spherical harmonics, we refer to [SW, Ch. IV].

1.2. Construction of polynomial frames on $\mathbb{S}^{d-1}$. Various nonstationary wavelets or frames have been constructed on the sphere by many authors (see ADS, [AV], DDSW], FGS, G], MNPW], [NW]). Although each of these wavelets or frames has its own advantage, to the best of our knowledge none of them has been shown useful in the characterization of classic function spaces on the sphere. In this subsection, we will construct a polynomial frame on $\mathbb{S}^{d-1}$, for which many function spaces on the sphere $\mathbb{S}^{d-1}$, such as $L^{p}, H^{p}$ and Besov spaces, can be characterized in terms of the coefficients in the wavelet decompositions.

Our construction is motivated by the approach taken in the pioneering work of MNPW, where the authors used positive cubature formulae to introduce a class of polynomial frames suitable for analyzing data on the sphere. It is based on the following theorem.

Theorem A. There exists a constant $\gamma>0$ depending only on d such that for any integer $N>0$ and any finite set $\left\{\xi_{k}\right\}_{k \in \Omega}$ of distinct points $\xi_{k} \in \mathbb{S}^{d-1}$ satisfying

$$
\min _{\substack{i, j \in \Omega \\ i \neq j}} d\left(\xi_{i}, \xi_{j}\right) \geq \gamma / N \text { and } \max _{x \in \mathbb{S}^{d-1}} \min _{j \in \Omega} d\left(x, \xi_{j}\right)<\gamma / N,
$$

there exists a set of numbers $0 \leq a_{N, k} \leq C_{d} N^{-(d-1)}, \quad k \in \Omega$, such that for any $f \in \Pi_{N}, 0<p \leq \infty$ and $t \geq 0$,

$$
\begin{aligned}
& \int_{\mathbb{S}^{d-1}} f(y) d \sigma(y)=\sum_{k \in \Omega} a_{N, k} f\left(\xi_{k}\right), \\
& \|f\|_{p} \asymp \begin{cases}\left(\frac{1}{N^{d-1}} \sum_{k \in \Omega}\left(N^{d-1} a_{N, k}\right)^{t}\left|f\left(\xi_{k}\right)\right|^{p}\right)^{\frac{1}{p}}, & \text { if } 0<p<\infty, \\
\max _{k \in \Omega}\left[\left(N^{d-1} a_{N, k}\right)^{t}\left|f\left(\xi_{k}\right)\right|\right], & \text { if } p=\infty,\end{cases}
\end{aligned}
$$


where the constants of equivalence depend only on $d$ and $p$ when $p$ is small, and in sequel, we employ the slight abuse of notation that $0^{0}=1$.

Theorem A under the restriction $t \leq \min \{p, 1\}$ was obtained in $\mathrm{BD}$, Theorem 3.1]. It will be shown in Section 5 that the restriction $t \leq \min \{p, 1\}$ is in fact not necessary.

An equality like (1.3) with nonnegative coefficients is called a positive cubature formula, while an equivalence like (1.4) is called a Marcinkiewicz-Zygmund (MZ) type inequality. It should be pointed out that positive cubature formulae and MZ inequalities for $1 \leq p \leq \infty$ based on function values at scattered sites on $\mathbb{S}^{d-1}$ were first established in the fundamental paper [MNW].

Now to each integer $j>0$ we assign a finite set $\left\{x_{j, k}: k \in \Lambda_{j}^{d}\right\}$ of distinct points $x_{j, k} \in \mathbb{S}^{d-1}$ satisfying

$$
\min _{\substack{k, k^{\prime} \in \Lambda_{j}^{d} \\ k \neq k^{\prime}}} d\left(x_{j, k}, x_{j, k^{\prime}}\right) \geq \frac{\gamma}{2^{j+4}} \text { and } \max _{x \in \mathbb{S}^{d-1}} \min _{k \in \Lambda_{j}^{d}} d\left(x, x_{j, k}\right)<\frac{\gamma}{2^{j+4}},
$$

with $\gamma$ as in Theorem A. Evidently, $\# \Lambda_{j}^{d} \asymp 2^{j(d-1)}$, and by Theorem A, there exists a set of numbers $0 \leq \lambda_{j, k} \leq C_{d} 2^{-j(d-1)}, k \in \Lambda_{j}^{d}$, such that for any $0<p \leq \infty$, $0 \leq t \leq \min \{p, 1\}$ and any $f \in \Pi_{2^{j+4}}$,

$$
\begin{gathered}
\int_{\mathbb{S}^{d-1}} f(y) d \sigma(y)=\sum_{k \in \Lambda_{j}^{d}} \lambda_{j, k} f\left(x_{j, k}\right), \\
\|f\|_{p} \asymp \begin{cases}\left(\frac{1}{2^{j(d-1)}} \sum_{k \in \Lambda_{j}^{d}}\left(2^{j(d-1)} \lambda_{j, k}\right)^{t}\left|f\left(x_{j, k}\right)\right|^{p}\right)^{\frac{1}{p}}, & \text { if } 0<p<\infty, \\
\max _{k \in \Lambda_{j}^{d}}\left[\left(2^{j(d-1)} \lambda_{j, k}\right)^{t}\left|f\left(x_{j, k}\right)\right|\right], & \text { if } p=\infty,\end{cases}
\end{gathered}
$$

where the constants of equivalence depend only on $d$ and $p$ when $p$ is small. For convenience, we also set $\Lambda_{0}^{d}=\{0\}, \lambda_{0,0}=1$ and take $x_{0,0}$ to be any fixed point on $\mathbb{S}^{d-1}$.

Let $\phi$ be a nonnegative $C^{\infty}$-function on $\mathbb{R}$ supported in $\left\{x \in \mathbb{R}: \frac{1}{2} \leq|x| \leq 2\right\}$ and satisfying

$$
\sum_{j=-\infty}^{\infty}\left(\phi\left(2^{-j} x\right)\right)^{2}=1, \text { for all } x \neq 0 .
$$

Together with $\phi$ we define a sequence of functions

$$
\psi_{j, k}(x):=\sqrt{\lambda_{j, k}} G_{j}\left(x \cdot x_{j, k}\right), j \geq 0, k \in \Lambda_{j}^{d},
$$

where

$$
G_{0}(t)=1, \quad G_{j}(t)=\sum_{k=\left[2^{j-2}\right]}^{2^{j}} \phi\left(\frac{k}{2^{j-1}}\right) P_{k}(t), \quad t \in[-1,1], \quad j \geq 1,
$$

and $P_{k}(t)$ is defined by (1.2). We index these functions by the spherical caps $B\left(x_{j, k}, 2^{-j} \pi\right)$. Thus with $B=B\left(x_{j, k}, 2^{-j} \pi\right)$ we let

$$
\psi_{B}(x)=\psi_{j, k}(x)=\sqrt{\lambda_{j, k}} G_{j}\left(x \cdot x_{j, k}\right) .
$$


We also use the notation $\mathcal{B}_{j}$ to denote the set of spherical caps $B\left(x_{j, k}, 2^{-j} \pi\right)$ $\left(k \in \Lambda_{j}^{d}\right)$ and $\mathcal{B}$ to denote the union of the $\mathcal{B}_{j}, j \geq 0$. Thus, associated with each distribution $f$ on $\mathbb{S}^{d-1}$, there is a series $\sum_{B \in \mathcal{B}}\left\langle f, \psi_{B}\right\rangle \psi_{B}$, and moreover, for each spherical polynomial $f$, we have

$$
f(x)=\sum_{B \in \mathcal{B}}\left\langle f, \psi_{B}\right\rangle \psi_{B}(x),
$$

with only a finite number of nonzero coefficients $\left\langle f, \psi_{B}\right\rangle$, as can be easily verified. We will keep the above notations for the rest of the paper.

We have three purposes in this paper. First, we want to study the unconditional convergence of the series $\sum_{B \in \mathcal{B}}\left\langle f, \psi_{B}\right\rangle \psi_{B}(x)$. Second, we want to characterize classic function spaces on $\mathbb{S}^{d-1}$, such as $L^{p}, H^{p}$ and Besov spaces, using the coefficients $\left\langle f, \psi_{B}\right\rangle,(B \in \mathcal{B})$. Third, we wish to investigate a related nonlinear $m$-term approximation problem. Our main results will be summarized in the next subsection.

1.3. Summary of main results. To state our results in a unified manner, we identify $H^{\infty}\left(\mathbb{S}^{d-1}\right)$ with $C\left(\mathbb{S}^{d-1}\right)$ and note that (see $[\mathrm{C}]$ ) for $1<p<\infty$, the Hardy space $H^{p}\left(\mathbb{S}^{d-1}\right)$ coincides with the Lebesgue space $L^{p}\left(\mathbb{S}^{d-1}\right)$ and $\|f\|_{p} \asymp\|f\|_{H^{p}}$ with the constants of equivalence depending only on $d$ and $p$.

Our first result gives a characterization of the spaces $H^{p}, 0<p<\infty$.

Theorem 1.1. If $0<p<\infty$ and $f \in H^{p}\left(\mathbb{S}^{d-1}\right)$, then $\sum_{B \in \mathcal{B}}\left\langle f, \psi_{B}\right\rangle \psi_{B}$ converges unconditionally to $f$ in the $H^{p}$-metric, and moreover

$$
\|f\|_{H^{p}} \asymp\left\|\left(\sum_{B \in \mathcal{B}}\left|\left\langle f, \psi_{B}\right\rangle\right|^{2}\left|\psi_{B}\right|^{2}\right)^{\frac{1}{2}}\right\|_{p} \asymp\left\|\left(\sum_{B \in \mathcal{B}}\left|\left\langle f, \psi_{B}\right\rangle\right|^{2}|B|^{-1} \chi_{B}\right)^{\frac{1}{2}}\right\|_{p},
$$

with the constants of equivalence depending only on $d, p$ and $\phi$. In addition, if $0<p<\infty$ and $\left\{a_{B}\right\}_{B \in \mathcal{B}}$ is a sequence of complex numbers such that either $\left(\sum_{B \in \mathcal{B}}\left|a_{B}\right|^{2}\left|\psi_{B}\right|^{2}\right)^{\frac{1}{2}} \in L^{p}\left(\mathbb{S}^{d-1}\right)$ or $\left(\sum_{B \in \mathcal{B}}\left|a_{B}\right|^{2}|B|^{-1} \chi_{B}\right)^{\frac{1}{2}} \in L^{p}\left(\mathbb{S}^{d-1}\right)$, then $\sum_{B \in \mathcal{B}} a_{B} \psi_{B}$ converges unconditionally to some distribution $f$ in the $H^{p}$-metric, and moreover

$$
\|f\|_{H^{p}} \leq C_{1}\left\|\left(\sum_{B \in \mathcal{B}}\left|a_{B}\right|^{2}\left|\psi_{B}\right|^{2}\right)^{\frac{1}{2}}\right\|_{p} \leq C_{2}\left\|\left(\sum_{B \in \mathcal{B}}\left|a_{B}\right|^{2}|B|^{-1} \chi_{B}\right)^{\frac{1}{2}}\right\|_{p},
$$

with the constants $C_{1}$ and $C_{2}$ depending only on $d, p$ and $\phi$.

Our next result concerns a characterization of the Besov spaces. For $\alpha>0$ and $0<p, \tau \leq \infty$, we define

$$
|f|_{B_{\tau}^{\alpha}\left(H^{p}\right)}:=\left(\sum_{k=0}^{\infty}(k+1)^{\alpha \tau-1}\left(E_{k}(f)_{H^{p}}\right)^{\tau}\right)^{\frac{1}{\tau}}+|\langle f, 1\rangle|
$$

with the usual change when $\tau=\infty$, where

$$
E_{n}(f)_{H^{p}}:=\inf \left\{\|f-g\|_{H^{p}}: \quad g \in \Pi_{n}\right\}, \quad n \in \mathbb{Z}_{+},
$$

and define the Besov space $B_{\tau}^{\alpha}\left(H^{p}\right)$ to be a linear space of distributions on $\mathbb{S}^{d-1}$ endowed with the quasi-norm $|\cdot|_{B_{\tau}^{\alpha}\left(H^{p}\right)}$. We point out that Besov spaces on the sphere were introduced and investigated by Nikol'skii, Lizorkin and Rustamov in a series of papers (see $[\underline{R}$ and the references there). These spaces can be equivalently 
characterized using the K-functionals or moduli of smoothness on the sphere (see [R]).

Theorem 1.2. For $\alpha>0,0<p, \tau \leq \infty$ and $f \in B_{\tau}^{\alpha}\left(H^{p}\right)$, we have

$$
|f|_{B_{\tau}^{\alpha}\left(H^{p}\right)} \asymp\left(\sum_{j=0}^{\infty} 2^{-j(d-1) \tau\left(\frac{1}{p}-\frac{1}{2}-\frac{\alpha}{d-1}\right)}\left(\sum_{k \in \Lambda_{j}^{d}}\left|\left\langle f, \psi_{j, k}\right\rangle\right|^{p}\right)^{\frac{\tau}{p}}\right)^{\frac{1}{\tau}},
$$

with the usual change when $p=\infty$ or $\tau=\infty$, where the constants of equivalence depend only on $\alpha, \tau, p$ and $\phi$. In addition, if $\left\{a_{j, k}: j=1,2, \ldots, k \in \Lambda_{j}^{d}\right\}$ is a sequence of complex numbers such that

$$
\left(\sum_{j=0}^{\infty} 2^{-j(d-1) \tau\left(\frac{1}{p}-\frac{1}{2}-\frac{\alpha}{d-1}\right)}\left(\sum_{k \in \Lambda_{j}^{d}}\left|a_{j, k}\right|^{p}\right)^{\frac{\tau}{p}}<\infty,\right.
$$

then the series $\sum_{j=0}^{\infty} \sum_{k \in \Lambda_{j}^{d}} a_{j, k} \psi_{j, k}$ converges unconditionally to some $f \in B_{\tau}^{\alpha}\left(H^{p}\right)$ in the $H^{p}$-metric. Moreover,

$$
|f|_{B_{\tau}^{\alpha}\left(H^{p}\right)} \leq C_{p, \alpha, \tau, \phi}\left(\sum_{j=0}^{\infty} 2^{-j(d-1) \tau\left(\frac{1}{p}-\frac{1}{2}-\frac{\alpha}{d-1}\right)}\left(\sum_{k \in \Lambda_{j}^{d}}\left|a_{j, k}\right|^{p}\right)^{\frac{\tau}{p}}\right)^{\frac{1}{\tau}}
$$

with the usual change when $p=\infty$ or $\tau=\infty$.

Of particular interest are the spaces $B_{\tau}^{\alpha}\left(H^{\tau}\right)$, for which we have

$$
|f|_{B_{\tau}^{\alpha}\left(H^{\tau}\right)} \asymp\left(\sum_{B \in \mathcal{B}}\left|\left\langle f, \psi_{B}\right\rangle\right|^{\tau}|B|^{1-\frac{\tau}{2}-\frac{\alpha \tau}{d-1}}\right)^{\frac{1}{\tau}},
$$

with the usual change when $\tau=\infty$, due to Theorem 1.2.

We point out that for the usual Euclidean space $\mathbb{R}^{d}$, results similar to Theorems 1.1 and 1.2 can be found in [FJ], [HW, Chapter 7] and [DJP.

Finally, we state our results on nonlinear approximation. For $0<p \leq \infty, f \in H^{p}$ and an integer $n>0$, we denote by $\Gamma_{n} \equiv \Gamma_{n, f, p}$ a set of $n$ spherical caps $B \in \mathcal{B}$ such that

$$
\min _{B \in \Gamma_{n}}\left|\left\langle f, \psi_{B}\right\rangle\right||B|^{\frac{1}{p}-\frac{1}{2}} \geq \max _{B \in \mathcal{B} \backslash \Gamma_{n}}\left|\left\langle f, \psi_{B}\right\rangle\right||B|^{\frac{1}{p}-\frac{1}{2}},
$$

and define the greedy-type algorithm $G_{n}^{p}(f)$ by

$$
G_{n}^{p}(f)=\sum_{B \in \Gamma_{n}}\left\langle f, \psi_{B}\right\rangle \psi_{B}
$$

Such an algorithm is well defined, as was shown in [T1, Remark 1.1]. We refer to the impressive survey paper [T2] for the background information on the greedy algorithm.

Theorem 1.3. For $\alpha>0,0<p \leq \infty, \tau=\left(\frac{\alpha}{d-1}+\frac{1}{p}\right)^{-1}, f \in H^{p}$ and an integer $n>0$,

$$
\left\|f-G_{n}^{p}(f)\right\|_{H^{p}} \leq C_{p, \alpha, \phi} n^{-\frac{\alpha}{d-1}}|f|_{B_{\tau}^{\alpha}\left(H^{\tau}\right)} .
$$


Theorem 1.4. For $\alpha>0,0<p \leq \infty, \tau=\left(\frac{1}{p}+\frac{\alpha}{d-1}\right)^{-1}, f \in H^{p}$ and an integer $n>0$,

$$
\left\|G_{n}^{p}(f)\right\|_{B_{\tau}^{\alpha}\left(H^{\tau}\right)} \leq C_{p, \alpha, \phi} n^{\frac{\alpha}{d-1}}\|f\|_{H^{p}} .
$$

In addition, if $f \in H^{p}$ has a representation $f(x)=\sum_{B \in \mathcal{B}} a_{B} \psi_{B}(x)$ with at most $n$ nonzero coefficients $a_{B}$, then

$$
|f|_{B_{\tau}^{\alpha}\left(H^{\tau}\right)} \leq C_{p, \alpha, \phi} n^{\frac{\alpha}{d-1}}\left\|\max _{B \in \mathcal{B}}\left[\left|a_{B}\right||B|^{-\frac{1}{2}} \chi_{B}(\cdot)\right]\right\|_{p} .
$$

The inequality (1.13) is a direct theorem of approximation (the Jackson inequality), while the inequality (1.14) is an inverse theorem (the Bernstein inequality). Once (1.13) and (1.14) are established, then by the standard method (see [DP]), the following characterization result holds for $0<\beta<\alpha$ :

$$
\sum_{n=1}^{\infty}\left[2^{n \beta /(d-1)}\left\|f-G_{2^{n}}^{p}(f)\right\|_{H^{p}}\right]^{\tau}<\infty \Longleftrightarrow f \in B_{\tau}^{\beta}\left(H^{\tau}\right)
$$

where $f \in H^{p}, 0<p \leq \infty$ and $\tau=(\beta /(d-1)+1 / p)^{-1}$. For results on the nonlinear approximation associated with wavelet decomposition in $L^{p}\left(\mathbb{R}^{d}\right)(0<p \leq \infty)$, we refer to DJP, [DPY] and [Jia].

We organize this paper as follows. Section 2 contains three lemmas which will be used frequently in the proofs of our main results. We prove Theorem 1.1 in Section 3 for the case $1<p<\infty$, and in Section 4 for the case $0<p \leq 1$. The proof of Theorem 1.2 and those of Theorems 1.3 and 1.4 are given in Sections 5 and 6, respectively.

Finally, we point out that our paper does not give any effective algorithms for spherical wavelets. For results in this direction, we refer the reader to the papers SS1], SS2 and [KL. We also note that characterizations of function spaces using wavelets on stratified Lie groups had been considered in $[\mathrm{L}$. (The author would like to thank an anonymous referee very much for kindly pointing out these references ([SS1, SS2, KL, L1) to him.) For more recent work on spherical frames, we refer to the paper NPW] by F. J. Narcowich, P. Petrushev and J. D. Ward, and also the nice survey paper [MP] by H. N. Mhaskar and J. Prestin.

\section{Three Useful Lemmas}

This section contains three lemmas that will be useful in the proofs of the main results in this paper. The first two lemmas (Lemmas 2.1 and 2.2) are in essence known, while the last one (Lemma 2.3) is new and will be of fundamental importance.

For the statement of Lemma 2.1, we define, for $f \in \mathcal{S}^{\prime}\left(\mathbb{S}^{d-1}\right)$,

$$
\sigma_{j}(f)(x):=\left\langle f, G_{j}(x \cdot)\right\rangle, \quad x \in \mathbb{S}^{d-1}, \quad j=0,1,2, \ldots,
$$

where $G_{j}$ is defined by (1.8).

Lemma 2.1. For $1<p<\infty$ and $f \in L^{p}\left(\mathbb{S}^{d-1}\right)$,

$$
\left\|\left(\sum_{j=0}^{\infty}\left|\sigma_{j}(f)\right|^{2}\right)^{\frac{1}{2}}\right\|_{p} \leq C_{p}\|f\|_{p} .
$$


In addition, if $f$ is a spherical polynomial, then

$$
\sum_{j=0}^{\infty} \sigma_{j} \circ \sigma_{j}(f)(x)=f(x), \quad \forall x \in \mathbb{S}^{d-1},
$$

with only a finite number of nonzero terms.

Proof. The inequality (2.2) is a simple consequence of the well-known Hörmandertype multiplier theorem for spherical harmonics (see $[\underline{S}$, Theorem 2]), while the identity (2.3) follows directly from (1.7) and the definition.

To state our next lemma, we suppose $\varphi$ is a $C^{\infty}$-function on $[0, \infty)$ supported in $[0,2]$ and equal to a constant on $\left[0, \frac{1}{2}\right]$, and define

$$
K_{N, \varphi}(t):=\sum_{k=0}^{\infty} \varphi\left(\frac{k}{N}\right) P_{k}(t), \quad t \in[-1,1], \quad N=1,2, \ldots,
$$

with $P_{k}(t)$ as defined in (1.2). Then, with these notations, we have

Lemma 2.2. For $\theta \in[0, \pi]$ and any positive integer $\ell$,

$$
\left|K_{N, \varphi}^{(i)}(\cos \theta)\right| \leq C_{\varphi, \ell, i} N^{d-1+2 i} \min \left\{1,(N \theta)^{-\ell}\right\}, \quad i=0,1, \ldots, N=1,2, \ldots,
$$

where $K_{N, \varphi}^{(0)}(t)=K_{N, \varphi}(t), K_{N, \varphi}^{(i)}(t)=\left(\frac{d}{d t}\right)^{i}\left\{K_{N, \varphi}(t)\right\}, i \geq 1$.

We note that for the usual Cesàro kernel

$$
\sigma_{N}^{\delta}(t):=\sum_{k=0}^{N} \frac{\Gamma(N-k+\delta+1) \Gamma(N+1)}{\Gamma(N-k+1) \Gamma(N+\delta+1)} P_{k}(t)
$$

of order $\delta>d-1$ it is well known (see $[\mathrm{BC}]$ ) that

$$
\left|\sigma_{N}^{\delta}(\cos \theta)\right| \leq C_{\delta} N^{d-1} \min \left\{1,(N \theta)^{-d}\right\},
$$

and the order $(N \theta)^{-d}$ on the right-hand side of this last inequality cannot be further improved. The significance point of Lemma 2.2 is that the positive integer $\ell$ can be chosen as big as we like, which will play a very important role when we deal with the case $0<p<1$ in the later sections.

The proof of Lemma 2.2 is contained in [BD, Lemma 3.3].

The following lemma will be of fundamental importance in the proofs of our main results.

Lemma 2.3. Suppose $f$ is a spherical polynomial of degree at most $N$ (i.e., $f \in$ $\left.\Pi_{N}\right)$. Then for any $\beta>0$ and $x \in \mathbb{S}^{d-1}$,

$$
f_{\beta, N}^{*}(x) \leq C_{\beta}\left(M\left(|f|^{\frac{1}{\beta}}\right)(x)\right)^{\beta},
$$

where

$$
f_{\beta, N}^{*}(x):=\sup _{y \in \mathbb{S}^{d-1}} \frac{|f(y)|}{(1+N d(x, y))^{\beta(d-1)}} .
$$

Proof. Let $\eta$ be a $C^{\infty}$-function on $[0, \infty)$ supported in $[0,2]$ and equal to 1 on $[0,1]$ and let $K_{N, \eta}$ be defined by (2.4) with $\varphi$ replaced by $\eta$. Then, clearly, for $f \in \Pi_{N}$,

$$
f(y)-f(z)=\int_{\mathbb{S}^{d-1}} f(u)\left(K_{N, \eta}(y \cdot u)-K_{N, \eta}(z \cdot u)\right) d \sigma(u), \quad y, z \in \mathbb{S}^{d-1} .
$$


For simplicity, we set, for $y, u \in \mathbb{S}^{d-1}$ and $\delta \in\left(0, \frac{1}{4}\right)$,

$$
A_{N, \delta}(y, u):=\max _{z \in B\left(y, \frac{\delta}{N}\right)}\left|K_{N, \eta}(y \cdot u)-K_{N, \eta}(z \cdot u)\right| .
$$

Then, using Lemma 2.2 with $i=0,1$, it is easy to verify that for any integer $\ell>0$,

$$
A_{N, \delta}(y, u) \leq C_{\ell, \eta} \begin{cases}N^{d-1}, & \text { if } \theta \in\left[0, \frac{4 \delta}{N}\right], \\ \delta N^{d-1} \min \left\{1,(N \theta)^{-\ell}\right\}, & \text { if } \theta \in\left[\frac{4 \delta}{N}, \pi\right],\end{cases}
$$

where $\theta=d(y, u)$. Thus, using (2.7) and (2.8) with $\ell=[(\beta+1)(d-1)]+2$, we obtain that for $x, y \in \mathbb{S}^{d-1}$ and $\delta \in\left(0, \frac{1}{4}\right)$,

$$
\begin{aligned}
& \sup _{z \in B\left(y, \frac{\delta}{N}\right)} \frac{|f(y)-f(z)|}{(1+N d(x, y))^{\beta(d-1)}} \\
& \leq f_{\beta, N}^{*}(x) \int_{\mathbb{S}^{d-1}}\left(\frac{1+N d(x, u)}{1+N d(x, y)}\right)^{\beta(d-1)} A_{N, \delta}(y, u) d \sigma(u) \\
& \leq 2^{\beta(d-1)} f_{\beta, N}^{*}(x)\left[\int_{\mathbb{S}^{d-1}} A_{N, \delta}(y, u) d \sigma(u)\right. \\
& \left.\quad+\int_{\mathbb{S}^{d-1}}(1+N d(y, u))^{\beta(d-1)} A_{N, \delta}(y, u) d \sigma(u)\right] \\
& \leq C_{\eta, \beta} \delta f_{\beta, N}^{*}(x) .
\end{aligned}
$$

This implies that for any $x, y \in \mathbb{S}^{d-1}$ and $\delta \in\left(0, \frac{1}{4}\right)$,

$$
\begin{aligned}
& \frac{|f(y)|^{\frac{1}{\beta}}}{(1+N d(x, y))^{d-1}} \leq \frac{2^{\frac{1}{\beta}} \inf _{z \in B\left(y, \frac{\delta}{N}\right)}|f(z)|^{\frac{1}{\beta}}}{(1+N d(x, y))^{d-1}}+\left(2 C_{\eta, \beta} \delta f_{\beta, N}^{*}(x)\right)^{\frac{1}{\beta}} \\
\leq & \frac{C_{\beta}\left(\frac{N}{\delta}\right)^{d-1}}{(1+N d(x, y))^{d-1}} \int_{B\left(y, \frac{\delta}{N}\right)}|f(z)|^{\frac{1}{\beta}} d \sigma(z)+\left(2 C_{\eta, \beta} \delta f_{\beta, N}^{*}(x)\right)^{\frac{1}{\beta}} \\
\leq & C_{\beta} \delta^{-(d-1)} M\left(|f|^{\frac{1}{\beta}}\right)(x)+\left(2 C_{\eta, \beta} \delta f_{\beta, N}^{*}(x)\right)^{\frac{1}{\beta}} .
\end{aligned}
$$

So, taking the supremum over all $y \in \mathbb{S}^{d-1}$, we deduce

$$
f_{\beta, N}^{*}(x) \leq C_{\beta}^{\prime} \delta^{-(d-1) \beta}\left(M\left(|f|^{\frac{1}{\beta}}\right)(x)\right)^{\beta}+2^{\beta+1} C_{\eta, \beta} \delta f_{\beta, N}^{*}(x),
$$

which implies (2.5) by taking $\delta=\left(2^{\beta+2} C_{\eta, \beta}\right)^{-1}$. This completes the proof.

\section{Proof of Theorem 1.1 for $1<p<\infty$}

We need the following lemma, which means that for each $j \in \mathbb{Z}_{+}$and $k \in \Lambda_{j}^{d}$ the function $\psi_{j, k}$ is highly localized in the spherical cap $B\left(x_{j, k}, 2^{-j-1}\right)$.

Lemma 3.1. For any $r>0, x \in \mathbb{S}^{d-1}, j \in \mathbb{Z}_{+}$and $k \in \Lambda_{j}^{d}$,

$$
g_{j, k}(x) \leq C_{d, \phi}\left|\psi_{j, k}(x)\right| \leq C_{d, \phi, r}\left(M\left(\left|g_{j, k}\right|^{r}\right)(x)\right)^{\frac{1}{r}},
$$

where $g_{j, k}(x)=2^{j(d-1)} \sqrt{\lambda_{j, k}} \chi_{B\left(x_{j, k}, 2^{-j-1}\right)}(x)$.

Proof. When $j=0$, Lemma 3.1 is trivial. So we may assume $j>0$. Recall that for $j>0$,

$$
\psi_{j, k}(x)=\sqrt{\lambda_{j, k}} G_{j}\left(x \cdot x_{j, k}\right)=\sqrt{\lambda_{j, k}} \sum_{v=0}^{2^{j}} \phi\left(\frac{v}{2^{j-1}}\right) P_{v}\left(x \cdot x_{j, k}\right),
$$


where $\phi$ is a nonnegative $C^{\infty}$-function supported in $\left\{x \in \mathbb{R}: \frac{1}{2} \leq|x| \leq 2\right\}$ and satisfying (1.7), and $P_{v}$ is defined by (1.2). Since (see [Sz, 7.32.2)]) $\max _{t \in[0, \pi]}\left|P_{v}(\cos t)\right|=$ $P_{v}(1) \asymp v^{d-2}$, it follows that $\max _{t \in[0, \pi]}\left|G_{j}(\cos t)\right|=G_{j}(1) \asymp 2^{j(d-1)}$. Hence, using Bernstein's inequality for trigonometric polynomials, we obtain that for $t \in\left[0, \frac{1}{2^{j+1}}\right]$,

$$
\sqrt{\lambda_{j, k}} G_{j}(\cos t) \geq \frac{1}{2} \sqrt{\lambda_{j, k}} G_{j}(1) \asymp 2^{j(d-1)} \sqrt{\lambda_{j, k}},
$$

and the inequality $g_{j, k}(x) \leq C_{d, \phi}\left|\psi_{j, k}(x)\right|$ then follows.

For the proof of the inequality

$$
\left|\psi_{j, k}(x)\right| \leq C_{d, \phi, r}\left(M\left(\left|g_{j, k}\right|^{r}\right)(x)\right)^{\frac{1}{r}},
$$

we use Lemma 2.2 to obtain that

$$
\left|\psi_{j, k}(x)\right| \leq C_{d, \phi, r}{\sqrt{\lambda_{j, k}}}^{j(d-1)} \min \left\{1,\left(2^{j} \theta\right)^{-\frac{d-1}{r}}\right\},
$$

where $\theta=d\left(x, x_{j, k}\right)$. Therefore, if $0 \leq \theta=d\left(x, x_{j, k}\right) \leq 2^{-(j+1)}$, then since $B\left(x_{j, k}, 2^{-j-1}\right) \subset B\left(x, 2^{-j}\right)$,

$$
\begin{aligned}
\left|\psi_{j, k}(x)\right| & \leq C_{d, \phi, r} \sqrt{\lambda_{j, k}} 2^{j(d-1)}\left(\frac{1}{\left|B\left(x, 2^{-j}\right)\right|} \int_{B\left(x, 2^{-j}\right)} \chi_{B\left(x_{j, k}, 2^{-j-1}\right)}(y) d \sigma(y)\right)^{\frac{1}{r}} \\
& \leq C_{d, \phi, r}\left(M\left(\left|g_{j, k}\right|^{r}\right)(x)\right)^{\frac{1}{r}}
\end{aligned}
$$

and if $\theta=d\left(x, x_{j, k}\right)>2^{-j-1}$, then since $B\left(x_{j, k}, 2^{-j-1}\right) \subset B(x, 2 \theta)$,

$$
\begin{aligned}
\left|\psi_{j, k}(x)\right| & \leq C_{d, \phi, r} \sqrt{\lambda_{j, k}} 2^{j(d-1)}\left(2^{j} \theta\right)^{-(d-1) / r} \\
& \leq C_{d, \phi, r} \sqrt{\lambda_{j, k}} 2^{j(d-1)}\left(\frac{1}{|B(x, 2 \theta)|} \int_{B(x, 2 \theta)} \chi_{B\left(x_{j, k}, 2^{-j-1}\right)}(y) d \sigma(y)\right)^{\frac{1}{r}} \\
& \leq C_{d, \phi, r}\left(M\left(\left|g_{j, k}\right|^{r}\right)(x)\right)^{\frac{1}{r}} .
\end{aligned}
$$

In either case, we have the desired estimate (3.2), and the proof is therefore complete.

Proof of Theorem 1.1 for $1<p<\infty$. First, we show that for any sequence $\left\{a_{B}\right\}_{B \in \mathcal{B}}$ of complex numbers,

$$
\left\|\left(\sum_{B \in \mathcal{B}}\left|a_{B}\right|^{2}\left|\psi_{B}\right|^{2}\right)^{\frac{1}{2}}\right\|_{p} \leq C_{p, d, \phi}\left\|\left(\sum_{B \in \mathcal{B}}\left|a_{B}\right|^{2}|B|^{-1} \chi_{B}\right)^{\frac{1}{2}}\right\|_{p} .
$$

Indeed, since $0 \leq \lambda_{j, k} \leq C_{d} 2^{-j(d-1)}$, by Lemma 3.1 applied to $r=1$ it follows that for any $B \in \mathcal{B},\left|\psi_{B}\right| \leq C_{d, \phi}|B|^{-\frac{1}{2}} M\left(\chi_{B}\right)$, which together with the well-known Fefferman-Stein inequality implies the inequality (3.3).

Second, we show that for any sequence $\left\{a_{B}\right\}_{B \in \mathcal{B}}$ of complex numbers and any finite subset $\mathcal{F} \subset \mathcal{B}$,

$$
\left\|\sum_{B \in \mathcal{F}} a_{B} \psi_{B}\right\|_{p} \leq C_{p, d, \phi}\left\|\left(\sum_{B \in \mathcal{F}}\left|a_{B}\right|^{2}\left|\psi_{B}\right|^{2}\right)^{\frac{1}{2}}\right\|_{p}
$$


Once (3.4) is proved, then by a standard argument, we deduce that the series $\sum_{B \in \mathcal{B}} a_{B} \psi_{B}$ converges unconditionally in the space $L^{p}$ provided that

$$
\left(\sum_{B \in \mathcal{B}}\left|a_{B}\right|^{2}\left|\psi_{B}\right|^{2}\right)^{\frac{1}{2}} \in L^{p}
$$

This together with (3.3) will imply the second assertion of Theorem 1.1 in the case $1<p<\infty$.

For the proof of (3.4), we define

$$
\sigma_{j}^{* *}(f)(x)=\sup _{y \in \mathbb{S}^{d-1}} \frac{\left|\sigma_{j}(f)(y)\right|}{\left(1+2^{j} d(x, y)\right)^{d-1}},
$$

where $\sigma_{j}(f)$ is defined by (2.1). For $j \in \mathbb{Z}_{+}$and $k \in \Lambda_{j}^{d}$, we write $B_{j, k}=$ $B\left(x_{j, k}, 2^{-j} \pi\right), B_{j, k}^{\prime}=B\left(x_{j, k}, 2^{-j-1}\right)$, and we define $a_{j, k}^{\prime}=a_{B_{j, k}}$ if $B_{j, k} \in \mathcal{F}$, and $=0$ otherwise. Also, we set $h=\sum_{B \in \mathcal{F}} a_{B} \psi_{B}$. Let $g \in L^{p^{\prime}}$ be such that $\|g\|_{p^{\prime}}=1$ and $\|h\|_{p}=\int_{\mathbb{S}^{d-1}} f(x) g(x) d \sigma(x)$, where $p^{\prime}=\frac{p}{p-1}$. We observe that

$$
\left|\left\langle g, \psi_{j, k}\right\rangle\right|=\sqrt{\lambda_{j, k}}\left|\sigma_{j}(g)\left(x_{j, k}\right)\right| \leq C_{d} 2^{j(d-1)} \sqrt{\lambda_{j, k}} \int_{B_{j, k}^{\prime}} \sigma_{j}^{* *}(g)(x) d \sigma(x) .
$$

Therefore

$$
\begin{aligned}
\|h\|_{p} & =\sum_{j=0}^{\infty} \sum_{k \in \Lambda_{j}^{d}} a_{j, k}^{\prime}\left\langle\psi_{j, k}, g\right\rangle \leq C_{d} \sum_{j, k}\left|a_{j, k}^{\prime}\right| \sqrt{\lambda_{j, k}} 2^{j(d-1)} \int_{B_{j, k}^{\prime}} \sigma_{j}^{* *}(g)(x) d x \\
& \leq C_{d}\left\|\left(\sum_{j, k}\left|a_{j, k}^{\prime}\right|^{2} \lambda_{j, k} 2^{2 j(d-1)} \chi_{B_{j, k}^{\prime}}\right)^{\frac{1}{2}}\right\|\left\|_{p}\right\|\left(\sum_{j=0}^{\infty} \sum_{k \in \Lambda_{j}^{d}}\left|\sigma_{j}^{* *}(g)\right|^{2} \chi_{B_{j, k}^{\prime}}\right)^{\frac{1}{2}} \|_{p^{\prime}} \\
& \leq C_{d}\left\|\left(\sum_{j, k}\left|a_{j, k}^{\prime}\right|^{2} \lambda_{j, k} 2^{2 j(d-1)} \chi_{B_{j, k}^{\prime}}\right)^{\frac{1}{2}}\right\|\left\|_{p}\right\|\left(\sum_{j=0}^{\infty}\left|\sigma_{j}^{* *}(g)\right|^{2}\right)^{\frac{1}{2}} \|_{p^{\prime}} .
\end{aligned}
$$

Invoking Lemma 2.1, Lemma 2.3 with $b=1$ and $N=2^{j}$, and the Fefferman-Stein inequality, we deduce

$$
\left\|\left(\sum_{j=0}^{\infty}\left|\sigma_{j}^{* *}(g)\right|^{2}\right)^{\frac{1}{2}}\right\|_{p^{\prime}} \leq C_{p}\left\|\left(\sum_{j=0}^{\infty}\left|\sigma_{j}(g)\right|^{2}\right)^{\frac{1}{2}}\right\|_{p^{\prime}} \leq C_{p},
$$

while using the first inequality in Lemma 3.1, we have

$$
\left(\sum_{j, k}\left|a_{j, k}^{\prime}\right|^{2} \lambda_{j, k} 2^{2 j(d-1)} \chi_{B_{j, k}^{\prime}}\right)^{\frac{1}{2}} \leq C_{d, \phi}\left(\sum_{B \in \mathcal{F}}\left|a_{B}\right|^{2}\left|\psi_{B}\right|^{2}\right)^{\frac{1}{2}} .
$$

The desired inequality (3.4) then follows.

Finally, we show the first assertion of Theorem 1.1. We claim that it will suffice to prove that for $f \in L^{p}$,

$$
\left\|\left(\sum_{B \in \mathcal{B}}\left|\left\langle f, \psi_{B}\right\rangle\right|^{2}|B|^{-1} \chi_{B}\right)^{\frac{1}{2}}\right\|_{p} \leq C_{p, d, \phi}\|f\|_{p} .
$$


In fact, once (3.6) is proved, then by the second assertion of Theorem 1.1 we just proved, it follows that for $f \in L^{p}$, the series $\sum_{B \in \mathcal{B}}\left\langle f, \psi_{B}\right\rangle \psi_{B}$ is convergent unconditionally in $L^{p}$, and by the usual density argument we must have $f=\sum_{B \in \mathcal{B}}\left\langle f, \psi_{B}\right\rangle \psi_{B}$. This together with (3.3), (3.4) and (3.6) will imply the desired equivalences

$$
\|f\|_{p} \asymp\left\|\left(\sum_{B \in \mathcal{B}}\left|\left\langle f, \psi_{B}\right\rangle\right|^{2}\left|\psi_{B}\right|^{2}\right)^{\frac{1}{2}}\right\|_{p} \asymp\left\|\left(\sum_{B \in \mathcal{B}}\left|\left\langle f, \psi_{B}\right\rangle\right|^{2}|B|^{-1} \chi_{B}\right)^{\frac{1}{2}}\right\|_{p},
$$

and hence the first assertion of Theorem 1.1.

For the proof of (3.6), we recall that $\left\langle f, \psi_{j, k}\right\rangle=\sqrt{\lambda_{j, k}} \sigma_{j}(f)\left(x_{j, k}\right)$ and $0 \leq \lambda_{j, k} \leq$ $C_{d} 2^{-j(d-1)}$. Thus, by the definition, it is easy to verify that

$$
\sum_{j=0}^{\infty} \sum_{k \in \Lambda_{j}^{d}}\left|\left\langle f, \psi_{j, k}\right\rangle\right|^{2}\left|B_{j, k}\right|^{-1} \chi_{B_{j, k}}(x) \leq C_{d} \sum_{j=0}^{\infty}\left|\sigma_{j}^{* *}(f)(x)\right|^{2},
$$

where $\sigma_{j}^{* *}$ is defined by (3.5). The desired inequality (3.6) then follows by Lemma 2.1, Lemma 2.3 and the well-known Fefferman-Stein inequality. This completes the proof.

\section{Proof of Theorem 1.1 for $0<p \leq 1$}

We start with some basic definitions and facts related to the Hardy spaces $H^{p}\left(\mathbb{S}^{d-1}\right), 0<p<\infty$. For $x \in \mathbb{S}^{d-1}$ and $z \in B_{d}:=\left\{\left(z_{1}, \cdots, z_{d}\right) \in \mathbb{R}^{d}: z_{1}^{2}+\right.$ $\left.\cdots+z_{d}^{2}<1\right\}$, let $p_{z}(x)=c_{d} \frac{1-|z|^{2}}{|z-x|^{d}}$, where $c_{d}$ is chosen so that $\int_{\mathbb{S}^{d-1}} p_{z}(x) d \sigma(x)=1$ for all $z \in B_{d}$. $p_{z}$ belongs to $\mathcal{S}\left(\mathbb{S}^{d-1}\right)$ and is called the Poisson Kernel. Given a distribution $f \in \mathcal{S}^{\prime}\left(\mathbb{S}^{d-1}\right)$, we define its radial maximal function by

$$
P^{+} f(x)=\sup _{0 \leq r<1}\left|\left\langle f, p_{r x}\right\rangle\right|, \quad x \in \mathbb{S}^{d-1},
$$

and its $H^{p}$-quasi-norm (for a given $0<p<\infty$ ) by $\|f\|_{H^{p}}=\left\|P^{+} f\right\|_{p}$. For $0<$ $p<\infty$, the Hardy space $H^{p}\left(\mathbb{S}^{d-1}\right)$ is defined to be the space of all distributions $f \in \mathcal{S}^{\prime}\left(\mathbb{S}^{d-1}\right)$ with $\|f\|_{H^{p}}<\infty$. It is well known (see [C]) that if $1<p<\infty$, then the Hardy space $H^{p}$ coincides with the Lebesgue space $L^{p}$ and $\|f\|_{p} \asymp\|f\|_{H^{p}}$ with the constants of equivalence depending only on $p$ and $d$. We will restrict ourselves to the case $0<p \leq 1$ for the rest of this section.

For $0<p \leq 1,1 \leq q \leq \infty$ and a nonnegative integer $s$, a regular $(p, q, s)$-atom centered at a point $y \in \mathbb{S}^{d-1}$ is a function $a$ in $L^{q}\left(\mathbb{S}^{d-1}\right)$ satisfying the following three conditions:

(i) supp $a \subset B(y, r)$ for some $r>0$;

(ii) $\|a\|_{q} \leq r^{(d-1)\left(\frac{1}{q}-\frac{1}{p}\right)}$;

(iii) $\int_{\mathbb{S}^{d-1}} a(x) p(x) d \sigma(x)=0$ for all $p \in \Pi_{s}$.

An exceptional atom is a function $a$ in $L^{\infty}\left(\mathbb{S}^{d-1}\right)$ with $\|a\|_{\infty} \leq 1$. Then the well-known atomic decomposition theorem (see [C, Proposition 3.1]) states that if $0<p \leq 1,1<q \leq \infty, s \geq\left[(d-1)\left(\frac{1}{p}-1\right)\right]$ and $f \in H^{p}\left(\mathbb{S}^{d-1}\right)$, then there exist a sequence $\left\{c_{j}\right\}_{j=0}^{\infty}$ of complex numbers and a sequence $\left\{a_{j}\right\}_{j=0}^{\infty}$ of exceptional or 
regular $(p, q, s)$-atoms such that $\sum_{j=0}^{\infty} c_{j} a_{j}$ converges to $f$ in the space $H^{p}$ and

$$
\left(\sum_{j=0}^{\infty}\left|c_{j}\right|^{p}\right)^{\frac{1}{p}} \leq C_{p, d}\|f\|_{H^{p}} .
$$

Given $0<p \leq 1$, a $p$-molecule centered at a point $y \in \mathbb{S}^{d-1}$ is a function $m \in L^{2}\left(\mathbb{S}^{d-1}\right)$ satisfying the following two conditions:

(i') For some $r>0$ and $s>(d-1)\left(\frac{2}{p-1}\right)$,

$$
\left(\int_{\mathbb{S}^{d-1}}|m(x)|^{2}\left(1+\frac{d(x, y)}{r}\right)^{s} d \sigma(x)\right)^{\frac{1}{2}} \leq r^{-(d-1)\left(\frac{1}{p}-\frac{1}{2}\right)}
$$

$$
\int_{\mathbb{S}^{d-1}} m(x) p(x) d \sigma(x)=0, \quad \text { for all } p \in \Pi_{\left[(d-1)\left(\frac{1}{p}-1\right)\right]} .
$$

According to [C] p. 234], for $0<p \leq 1$, any $p$-molecule $m$ must satisfy $\|m\|_{H^{p}} \leq$ $C_{p, d}$.

For the proof of Theorem 1.1, we need the following.

Lemma 4.1. For $0<p \leq 1$ and $f \in H^{p}$,

$$
\left\|\left(\sum_{j=0}^{\infty}\left|\sigma_{j}(f)\right|^{2}\right)^{\frac{1}{2}}\right\|_{p} \leq C_{d, p, \phi}\|f\|_{H^{p}},
$$

where $\sigma_{j}$ is defined by (2.1).

Proof. Let $0<p \leq 1$ and $s=\left[(d-1)\left(\frac{1}{p}-1\right)\right]+2$. Since $\left(\sum_{j=0}^{\infty}\left|\sigma_{j}(f)\right|^{2}\right)^{\frac{1}{2}}$ is bounded on $L^{2}$, by the atomic decomposition theorem it will suffice to prove that for any regular $(p, \infty, s)$-atom $a$,

$$
\left\|\left(\sum_{j=0}^{\infty}\left|\sigma_{j}(a)\right|^{2}\right)^{\frac{1}{2}}\right\|_{p} \leq C_{p, d, \phi} .
$$

For the proof of (4.1), we suppose $a$ is a regular $(p, \infty, s)$-atom supported in $B\left(x_{0}, r\right)$ for some $x_{0} \in \mathbb{S}^{d-1}$ and $r \in\left(0, \frac{1}{4}\right)$. Then using Hölder's inequality, we have

$$
\begin{aligned}
& \left(\int_{B\left(x_{0}, 4 r\right)}\left(\sum_{j=0}^{\infty}\left|\sigma_{j}(a)(x)\right|^{2}\right)^{\frac{p}{2}} d \sigma(x)\right)^{\frac{1}{p}} \\
& \quad \leq\left(\int_{B\left(x_{0}, 4 r\right)} \sum_{j=0}^{\infty}\left|\sigma_{j}(a)(x)\right|^{2} d \sigma(x)\right)^{\frac{1}{2}}\left(\int_{B\left(x_{0}, 4 r\right)} d \sigma(x)\right)^{\frac{2-p}{2 p}} \\
& \leq C_{p, d, \phi} r^{\frac{(2-p)(d-1)}{2 p}}\|a\|_{2} \leq C_{p, d, \phi}^{\prime} .
\end{aligned}
$$


Thus, it remains to prove that

$$
\left(\int_{\mathbb{S}^{d-1} \backslash B\left(x_{0}, 4 r\right)}\left(\sum_{j=0}^{\infty}\left|\sigma_{j}(a)(x)\right|^{2}\right)^{\frac{p}{2}} d \sigma(x)\right)^{\frac{1}{p}} \leq C_{p, d, \phi} .
$$

To prove this last inequality, we claim that for $x \in \mathbb{S}^{d-1} \backslash B\left(x_{0}, 4 r\right)$ and $\ell=d+2 s+2$,

$$
\left|\sigma_{j}(a)(x)\right| \leq C_{p, d, \phi} 2^{j(d+2 s+1)} \theta^{s+1} r^{s-\frac{d-1}{p}+d} \min \left\{1,\left(2^{j} \theta\right)^{-\ell}\right\},
$$

where $\theta=d\left(x, x_{0}\right)$. Once the claim (4.3) is proved, then by straightforward calculation, we deduce that for $x \in \mathbb{S}^{d-1} \backslash B\left(x_{0}, 4 r\right)$,

$$
\left(\sum_{j=0}^{\infty}\left|\sigma_{j}(a)(x)\right|^{2}\right)^{\frac{1}{2}}=\left[\left(\sum_{2^{j} \theta \leq 1}+\sum_{2^{j} \theta>1}\right)\left|\sigma_{j}(a)(x)\right|^{2}\right]^{\frac{1}{2}} \leq C_{d, p, \phi} \theta^{-d-s} r^{s+d-\frac{d-1}{p}},
$$

with $\theta=d\left(x, x_{0}\right)$, from which the desired inequality (4.2) will follow.

Now the proof of Lemma 4.1 is reduced to the proof of the claim (4.3). We recall that

$$
\sigma_{j}(a)(x)=\int_{\mathbb{S}^{d-1}} a(y) G_{j}(x \cdot y) d \sigma(y) .
$$

Hence, by the definition of regular $(p, \infty, s)$-atom it follows that

$$
\left|\sigma_{j}(a)(x)\right| \leq r^{-\frac{d-1}{p}} \int_{B\left(x_{0}, r\right)}\left|G_{j}(x \cdot y)-\sum_{i=0}^{s} \frac{G_{j}^{(i)}\left(x \cdot x_{0}\right)}{i !}\left(x \cdot\left(y-x_{0}\right)\right)^{i}\right| d \sigma(y) .
$$

For $x \in \mathbb{S}^{d-1} \backslash B\left(x_{0}, 4 r\right)$ and $y \in B\left(x_{0}, r\right)$, we write $\theta=d\left(x, x_{0}\right)$ and $t=d(x, y)$. Then, evidently, $\frac{3 \theta}{4} \leq t \leq \frac{5 \theta}{4}$ and $\left|x \cdot\left(y-x_{0}\right)\right|=2\left|\sin \frac{\theta-t}{2} \sin \frac{\theta+t}{2}\right| \leq \frac{9}{8} \theta r$. Hence, by Lemma 2.2 it follows that for any $\ell>0, x \in \mathbb{S}^{d-1} \backslash B\left(x_{0}, 4 r\right)$ and $y \in B\left(x_{0}, r\right)$, $\left|G_{j}(x \cdot y)-\sum_{i=0}^{s} \frac{G_{j}^{(i)}\left(x \cdot x_{0}\right)}{i !}\left(x \cdot\left(y-x_{0}\right)\right)^{i}\right| \leq C_{p, d, \phi}(\theta r)^{s+1} 2^{j(d+2 s+1)} \min \left\{1,\left(2^{j} \theta\right)^{-\ell}\right\}$,

where $\theta=d\left(x, x_{0}\right)$. Substituting this last estimate into (4.4), we obtain that for $x \in \mathbb{S}^{d-1} \backslash B\left(x_{0}, 4 r\right)$,

$$
\begin{aligned}
\left|\sigma_{j}(a)(x)\right| & \leq C_{p, d, \phi} \int_{B\left(x_{0}, r\right)} r^{-\frac{d-1}{p}}(\theta r)^{s+1} 2^{j(d+2 s+1)} \min \left\{1,\left(2^{j} \theta\right)^{-\ell}\right\} d \sigma(y) \\
& \leq C_{p, d, \phi} 2^{j(d+2 s+1)} \theta^{s+1} r^{s-\frac{d-1}{p}+d} \min \left\{1,\left(2^{j} \theta\right)^{-\ell}\right\},
\end{aligned}
$$

proving the claim (4.3). This completes the proof.

Proof of Theorem 1.1 for $0<p \leq 1$. Following the proof in the last section, we need only verify the following three assertions in the case $0<p \leq 1$ :

(a) For any sequence $\left\{a_{B}\right\}_{B \in \mathcal{B}}$ of complex numbers,

$$
\left\|\left(\sum_{B \in \mathcal{B}}\left|a_{B}\right|^{2}\left|\psi_{B}\right|^{2}\right)^{\frac{1}{2}}\right\|_{p} \leq C_{p, d}\left\|\left(\sum_{B \in \mathcal{B}}\left|a_{B}\right|^{2}|B|^{-1} \chi_{B}\right)^{\frac{1}{2}}\right\|_{p}
$$

(b) for $f \in H^{p}$,

$$
\left\|\left(\sum_{B \in \mathcal{B}}\left|\left\langle f, \psi_{B}\right\rangle\right|^{2}|B|^{-1} \chi_{B}\right)^{\frac{1}{2}}\right\|_{p} \leq C_{p, d}\|f\|_{H^{p}}
$$


(c) for any finite subset $\mathcal{F} \subset \mathcal{B}$ and any sequence $\left\{a_{B}\right\}_{B \in \mathcal{F}}$ of complex numbers,

$$
\left\|\sum_{B \in \mathcal{F}} a_{B} \psi_{B}\right\|_{H^{p}} \leq C_{p, d}\left\|\left(\sum_{B \in \mathcal{F}}\left|a_{B}\right|^{2}\left|\psi_{B}\right|^{2}\right)^{\frac{1}{2}}\right\|_{p} .
$$

Assertion (a) follows directly from the Fefferman-Stein inequality and the second inequality in Lemma 3.1 with $0<r<p$.

For the proof of assertion (b), we define for $\beta>0$ and $j \in \mathbb{Z}_{+}$,

$$
\sigma_{j, \beta}^{* *}(f)(x):=\sup _{y \in \mathbb{S}^{d-1}} \frac{\left|\sigma_{j}(f)(y)\right|}{\left(1+2^{j} d(x, y)\right)^{\beta(d-1)}} .
$$

Then by Lemma 2.3 applied to $N=2^{j}$, we have

$$
\sigma_{j, \beta}^{* *}(f)(x) \leq C_{d, \beta}\left(M\left(\left|\sigma_{j}(f)\right|^{\frac{1}{\beta}}\right)(x)\right)^{\beta}
$$

and hence, by Lemma 4.1 and the Fefferman-Stein inequality, we obtain that for $\beta>\frac{1}{p}$,

$$
\left\|\left(\sum_{j=0}^{\infty}\left|\sigma_{j, \beta}^{* *}(f)\right|^{2}\right)^{\frac{1}{2}}\right\|_{p} \leq C_{p, d, \beta}\left\|\left(\sum_{j=0}^{\infty}\left|\sigma_{j}(f)\right|^{2}\right)^{\frac{1}{2}}\right\|_{p} \leq C_{p, d, \beta}^{\prime}\|f\|_{H^{p}} .
$$

Now assertion (b) follows from (4.5) and the following inequality, which can be easily verified:

$$
\left(\sum_{B \in \mathcal{B}}\left|\left\langle f, \psi_{B}\right\rangle\right|^{2}|B|^{-1} \chi_{B}(x)\right)^{\frac{1}{2}} \leq C_{\beta, d}\left(\sum_{j=0}^{\infty}\left|\sigma_{j, \beta}^{* *}(f)(x)\right|^{2}\right)^{\frac{1}{2}}, \forall \beta>0, \forall x \in \mathbb{S}^{d-1} .
$$

It remains to prove assertion (c). For simplicity, we define $a_{B}=0$ for $B \in \mathcal{B} \backslash \mathcal{F}$, we denote by $\mathcal{D}$ the set of all spherical caps $B\left(x_{j, k}, 2^{-j-1}\right)\left(j \in \mathbb{Z}_{+}, k \in \Lambda_{j}^{d}\right)$, for each $j \in \mathbb{Z}_{+}, k \in \Lambda_{j}^{d}$, we write

$a_{j, k}=a_{B\left(x_{j, k}, 2^{-j-1}\right)}:=a_{B\left(x_{j, k}, 2^{-j} \pi\right)}, \quad \lambda_{B\left(x_{j, k}, 2^{-j-1}\right)}:=\lambda_{j, k}, \quad \psi_{B\left(x_{j, k}, 2^{-j-1}\right)}:=\psi_{j, k}$, and for $c>0, B=B(x, r)$, we write $c B:=B(x, c r)$.

First, we observe that for $0<p \leq 1$

$$
\left\|\sum_{\substack{2^{j} \leq 8(d-1)\left(\frac{1}{p}-1\right) \\ k \in \Lambda_{j}^{d}}} a_{j, k} \psi_{j, k}\right\|_{H^{p}} \leq C_{p, d}\left\|\left(\sum_{\substack{2^{j} \leq 8(d-1)\left(\frac{1}{p}-1\right) \\ k \in \Lambda_{j}^{d}}}\left|a_{j, k}\right|^{2}\left|\psi_{j, k}\right|^{2}\right)^{\frac{1}{2}}\right\|_{p},
$$

since any two quasi-norms on a finite-dimensional linear space are equivalent. Thus, without loss of generality, we may assume

$$
a_{j, k}=0, \text { for all } 2^{j} \leq 8(d-1)\left(\frac{1}{p}-1\right) \text { and } k \in \Lambda_{j}^{d} .
$$

Set $W(x):=\left(\sum_{B \in \mathcal{D}}\left|a_{B}\right|^{2} \lambda_{B}|B|^{-2} \chi_{B}(x)\right)^{\frac{1}{2}}$. Then by the first inequality in Lemma $3.1, W(x) \leq C_{d}\left(\sum_{B \in \mathcal{B}}\left|a_{B}\right|^{2}\left|\psi_{B}(x)\right|^{2}\right)^{\frac{1}{2}}$. Thus, it suffices to prove

$$
\left\|\sum_{B \in \mathcal{B}} a_{B} \psi_{B}\right\|_{H^{p}} \leq C_{p, d}\|W\|_{p}
$$


For the proof of this last inequality, for an integer $k \in \mathbb{Z}$ we let

$$
\Omega_{k}=\left\{x \in \mathbb{S}^{d-1}: W(x) \geq 2^{k}\right\}, \quad \mathcal{A}_{k}=\left\{B \in \mathcal{D}: \quad\left|B \cap \Omega_{k}\right| \geq \frac{1}{2}|B|\right\}
$$

and let $\mathcal{D}_{k}=\mathcal{A}_{k} \backslash \mathcal{A}_{k+1}$. Then summation by parts yields

$$
\left(\sum_{k \in \mathbb{Z}} 2^{k p}\left|\Omega_{k}\right|\right)^{\frac{1}{p}} \leq C_{p}\|W\|_{p}
$$

For each integer $k \in \mathbb{Z}$, we choose a subset $\left\{B_{k}^{i}: \quad i \in \Gamma_{k}\right\}$ of $\mathcal{D}_{k}$ such that $B_{k}^{i} \cap B_{k}^{j}=\emptyset$ if $i, j \in \Gamma_{k}$ and $i \neq j$, and $\bigcup_{B \in \mathcal{D}_{k}} B \subset \bigcup_{i \in \Gamma_{k}} 2 B_{k}^{i}$. (The existence of such a subset $\left\{B_{k}^{i}: \quad i \in \Gamma_{k}\right\}$ is easy to verify.) Now for $k \in \mathbb{Z}$ and $i \in \Gamma_{k}$, we define

$$
b(k, i):=\left|B_{k}^{i}\right|^{\frac{1}{p}-\frac{1}{2}}\left(\sum_{\substack{B \in \mathcal{D}_{k} \\ B \subset 2 B_{k}^{i}}} \lambda_{B}|B|^{-1}\left|a_{B}\right|^{2}\right)^{\frac{1}{2}}
$$

and

$$
m_{k, i}(x):= \begin{cases}b(k, i)^{-1} \sum_{\substack{B \in \mathcal{D}_{k} \\ B \subset 2 B_{k}^{i}}} a_{B} \psi_{B}(x), & \text { if } b(k, i) \neq 0, \\ 0, & \text { if } b(k, i)=0 .\end{cases}
$$

Then it's easily seen that $\sum_{B \in \mathcal{D}} a_{B} \psi_{B}=\sum_{k \in \mathbb{Z}} \sum_{i \in \Gamma_{k}} b(k, i) m_{k, i}$. Therefore, for the proof of (4.7), it is sufficient to show that each $m_{k, i}$ is a $p$-molecule up to an absolute constant and

$$
\left(\sum_{k \in \mathbb{Z}} \sum_{i \in \Gamma_{k}}|b(k, i)|^{p}\right)^{\frac{1}{p}} \leq C_{p, d}\|W\|_{p} .
$$

Since the spaces $\left\{\mathcal{H}_{k}\right\}_{k=0}^{\infty}$ (of spherical harmonics) are mutually orthogonal, it follows by the assumption (4.6) that

$$
\int_{\mathbb{S}^{d-1}} m_{k, i}(x) p(x) d \sigma(x)=0, \quad \forall p \in \Pi_{\left[(d-1)\left(\frac{1}{p}-1\right)\right]} .
$$

Thus, to show that $m_{k, i}$ is a $p$-molecule up to an absolute constant, it suffices to prove that for any $s>0$,

$$
\left(\int_{\mathbb{S}^{d-1}}\left|m_{k, i}(x)\right|^{2}\left(1+\frac{d(x, z)}{r}\right)^{s} d x\right)^{\frac{1}{2}} \leq C_{p, d} r^{(d-1)\left(\frac{1}{2}-\frac{1}{p}\right)}
$$

where $z \equiv z_{k, i}$ denotes the center of $B_{k}^{i}$, and $r \equiv r_{k, i}$ denotes the radius of $B_{k}^{i}$. In fact, since

$$
b(k, i)\left\|m_{k, i}\right\|_{2} \leq C_{p, d}\left(\sum_{\substack{B \in \mathcal{D}_{k} \\ B \subset 2 B_{k}^{i}}}\left|a_{B}\right|^{2} \lambda_{B}|B|^{-1}\right)^{\frac{1}{2}}=b(k, i)\left|B_{k}^{i}\right|^{\frac{1}{2}-\frac{1}{p}},
$$

it follows that

$$
\left\|m_{k, i}\right\|_{2} \leq C_{p, d} r^{(d-1)\left(\frac{1}{2}-\frac{1}{p}\right)}
$$


However, on the other hand, for each $B \in \mathcal{D}_{k}$ with $B \subset 2 B_{k}^{i}$, and each $x \in$ $\mathbb{S}^{d-1} \backslash 4 B_{k}^{i}$, applying Lemma 2.2 , we obtain

$$
\left.\left|\psi_{B}(x) \leq C_{d, \ell}\right| B\right|^{\frac{\ell}{d-1}-1} \sqrt{\lambda_{B}}(d(x, z))^{-\ell}, \quad \forall \ell>\frac{s+d-1}{2} .
$$

Hence,

$$
\begin{aligned}
\left|\sum_{\substack{B \in \mathcal{D}_{k} \\
B \subset 2 B_{k}^{i}}} a_{B} \psi_{B}(x)\right|^{2} & \leq C_{d, \ell}\left(\sum_{\substack{B \in \mathcal{D}_{k} \\
B \subset 2 B_{k}^{i}}} \lambda_{B}|B|^{-1}\left|a_{B}\right|^{2}\right)\left(\sum_{\substack{B \in \mathcal{D}_{k} \\
B \subset 2 B_{k}^{i}}}|B|^{-1+\frac{2 \ell}{d-1}}\right)(d(x, z))^{-2 \ell} \\
& \leq C_{d, \ell}(b(k, i))^{2} r^{2 \ell-\frac{2(d-1)}{p}}(d(x, z))^{-2 \ell}, \quad \forall \ell>\frac{s+d-1}{2},
\end{aligned}
$$

which implies

$$
\left|m_{k, i}(x)\right|^{2} \leq C_{d, \ell} r^{2 \ell-\frac{2(d-1)}{p}}(d(x, z))^{-2 \ell}, \quad \forall \ell>\frac{s+d-1}{2} .
$$

We then deduce by a straightforward calculation that

$$
\left(\int_{d(x, z) \geq 4 r}\left|m_{k, i}(x)\right|^{2}\left(1+\frac{d(x, z)}{r}\right)^{s} d \sigma(x)\right)^{\frac{1}{2}} \leq C_{p, d} r^{(d-1)\left(\frac{1}{2}-\frac{1}{p}\right)},
$$

which together with (4.11) implies (4.10).

It remains to prove (4.9). We observe that

$$
\begin{aligned}
\sum_{\substack{B \in \mathcal{D}_{k} \\
B \subset 2 B_{k}^{i}}} \lambda_{B}|B|^{-1}\left|a_{B}\right|^{2} & \leq 2 \sum_{\substack{B \in \mathcal{D}_{k} \\
B \subset 2 B_{k}^{i}}}\left|a_{B}\right|^{2}|B|^{-2} \lambda_{B}\left|B \cap \Omega_{k+1}^{c}\right| \\
& \leq 2 \int_{\left(2 B_{k}^{i}\right) \cap \Omega_{k+1}^{c}}(W(x))^{2} d \sigma(x) \leq 2^{2 k+d+2}\left|B_{k}^{i}\right| .
\end{aligned}
$$

Thus, by the definition,

$$
b(k, i) \leq 2^{(d+2) / 2} 2^{k}\left|B_{k}^{i}\right|^{\frac{1}{p}} \quad\left(k \in \mathbb{Z}, i \in \Gamma_{k}\right),
$$

which implies

$$
\sum_{k, i}|b(k, i)|^{p} \leq 2^{(d+2) p / 2} \sum_{k \in \mathbb{Z}} 2^{k p} \sum_{i \in \Gamma_{k}}\left|B_{k}^{i}\right| \leq 2^{(d+2) p / 2} \sum_{k \in \mathbb{Z}} 2^{k p}\left|\Omega_{k}\right|
$$

since $\left\{B_{k}^{i}\right\}_{i \in \Gamma_{k}}$ is a sequence of mutually disjoint subsets of $\Omega_{k}$. This, combined with (4.8) gives (4.9), and therefore completes the proof.

\section{Proof of Theorem 1.2}

Recall that $\sigma_{j}$ is defined by (2.1). We need the following.

Lemma 5.1. For $\alpha>0$ and $0<p, \tau \leq \infty$,

$$
|f|_{B_{\tau}^{\alpha}\left(H^{p}\right)} \asymp\left(\sum_{j=0}^{\infty} 2^{j \alpha \tau}\left\|\sigma_{j} \circ \sigma_{j}(f)\right\|_{H^{p}}^{\tau}\right)^{\frac{1}{\tau}} \asymp\left(\sum_{j=0}^{\infty} 2^{j \alpha \tau}\left\|\sigma_{j}(f)\right\|_{H^{p}}^{\tau}\right)^{\frac{1}{\tau}},
$$

with the usual change when $\tau=\infty$, where the constants of equivalence depend only on $p, d, \alpha, \tau$ and $\phi$. 
Proof. By the definition, it is easily seen that the series $\sum_{j=0}^{\infty} \sigma_{j} \circ \sigma_{j}(f)$ converges to $f$ in the space $H^{p}$ and for each $k \in \mathbb{Z}_{+}, \sum_{j=0}^{k} \sigma_{j} \circ \sigma_{j}(f) \in \Pi_{2^{k}-1}$. Thus, for each $k \in \mathbb{Z}_{+}$and $f \in H^{p}$

$$
E_{2^{k}-1}(f)_{H^{p}} \leq\left\|\sum_{j=k+1}^{\infty} \sigma_{j} \circ \sigma_{j}(f)\right\|_{H^{p}} \leq\left(\sum_{j=k+1}^{\infty}\left\|\sigma_{j} \circ \sigma_{j}(f)\right\|_{H^{p}}^{q}\right)^{\frac{1}{q}},
$$

where $q=\min \{p, 1\}$. Since the operators $\sigma_{j}, j \in \mathbb{Z}_{+}$, are uniformly bounded on $H^{p}$, by the definition and Hardy-type inequality it follows that

$$
|f|_{B_{\tau}^{\alpha}\left(H^{p}\right)} \leq C_{\tau, \alpha}\left(\sum_{j=0}^{\infty} 2^{j \alpha \tau}\left\|\sigma_{j} \circ \sigma_{j}(f)\right\|_{H^{p}}^{\tau}\right)^{\frac{1}{\tau}} \leq C_{p, \tau, \alpha, \phi}\left(\sum_{j=0}^{\infty} 2^{j \alpha \tau}\left\|\sigma_{j}(f)\right\|_{H^{p}}^{\tau}\right)^{\frac{1}{\tau}}
$$

with the usual change when $\tau=\infty$.

On the other hand, noting that $\sigma_{j}(g)=0$ for any $g \in \Pi_{\left[2^{j-2}\right]}$ and $j \geq 1$, we obtain that for $j \geq 1$

$$
\left\|\sigma_{j}(f)\right\|_{H^{p}}=\inf _{\left.g \in \Pi_{\left[2^{j}-2\right.}\right]}\left\|\sigma_{j}(f-g)\right\|_{H^{p}} \leq C_{p, \phi} E_{\left[2^{j-2}\right]}(f)_{H^{p}} .
$$

This, together with the uniform boundedness of the operators $\sigma_{j}$ on $H^{p}$, implies the desired inverse inequalities

$$
\left(\sum_{j=0}^{\infty} 2^{j \alpha \tau}\left\|\sigma_{j} \circ \sigma_{j}(f)\right\|_{H^{p}}^{\tau}\right)^{\frac{1}{\tau}} \leq C_{p, \phi}\left(\sum_{j=0}^{\infty} 2^{j \alpha \tau}\left\|\sigma_{j}(f)\right\|_{H^{p}}^{\tau}\right)^{\frac{1}{\tau}} \leq C_{p, \phi}^{\prime}|f|_{B_{\tau}^{\alpha}\left(H^{p}\right)},
$$

with the usual change when $\tau=\infty$. This completes the proof.

As indicated in Section 1, the MZ-type inequality (1.4) in Theorem A under the restriction $0 \leq t \leq \min \{p, 1\}$ was proved in $\mathrm{BD}$, Theorem 3.1]. Our Lemma 5.2 below asserts that this same inequality, in fact, holds for $t>\min \{p, 1\}$ as well.

Lemma 5.2. Suppose that $\left\{\xi_{k}\right\}_{k \in \Omega}$ is a finite subset of $\mathbb{S}^{d-1}$ and $\left\{a_{N, k}\right\}_{k \in \Omega}$ is a sequence of nonnegative numbers smaller than $C_{d} N^{-(d-1)}$. Suppose further that the $M Z$ inequality (1.4) holds for all $f \in \Pi_{N}$ and $0 \leq t \leq \min \{p, 1\}$. Then we have, for any $0<p \leq \infty$, any $f \in \Pi_{N}$ and all $\alpha \geq 0$,

$$
\|f\|_{p} \asymp \begin{cases}\left(\frac{1}{N^{d-1}} \sum_{k \in \Omega}\left(N^{d-1} a_{N, k}\right)^{\alpha p}\left|f\left(\xi_{k}\right)\right|^{p}\right)^{\frac{1}{p}}, & \text { if } 0<p<\infty, \\ \max _{k \in \Omega}\left[\left(N^{d-1} a_{N, k}\right)^{\alpha}\left|f\left(\xi_{k}\right)\right|\right], & \text { if } p=\infty,\end{cases}
$$

with the constants of equivalence depending only on $d, p$ when $p$ is small, and $\alpha$ when $\alpha$ is big.

Proof. Since $0 \leq a_{N, k} \leq C_{d} N^{-(d-1)}$, by (1.4) with $t=0$ it follows that

$$
\left(\frac{1}{N^{d-1}} \sum_{k \in \Omega}\left(N^{d-1} a_{N, k}\right)^{\alpha p}\left|f\left(\xi_{k}\right)\right|^{p}\right)^{\frac{1}{p}} \leq C_{p, d, \alpha}\|f\|_{p}, \quad \forall \alpha \geq 0,
$$


with the usual change when $p=\infty$. To prove the inverse inequality, without loss of generality, we may assume $\alpha>1$. Set $\alpha_{0}=\min \{p, 1\}$. If $0<p<\infty$, then using (1.4) and Hölder's inequality, we have

$$
\begin{aligned}
\|f\|_{p}^{p} & \leq C_{p, d}\left(\frac{1}{N^{d-1}} \sum_{k \in \Omega}\left|f\left(\xi_{k}\right)\right|^{p}\left(N^{d-1} a_{N, k}\right)^{\alpha_{0}}\right) \\
& \leq C_{p, d}\left(\frac{1}{N^{d-1}} \sum_{k \in \Omega}\left|f\left(\xi_{k}\right)\right|^{p}\left(N^{d-1} a_{N, k}\right)^{\alpha p}\right)^{\frac{\alpha_{0}}{\alpha p}}\left(\frac{1}{N^{d-1}} \sum_{k \in \Omega}\left|f\left(\xi_{k}\right)\right|^{p}\right)^{1-\frac{\alpha_{0}}{\alpha p}} \\
& \leq C_{p, d}\left(\frac{1}{N^{d-1}} \sum_{k \in \Omega}\left|f\left(\xi_{k}\right)\right|^{p}\left(N^{d-1} a_{N, k}\right)^{\alpha p}\right)^{\frac{1}{p} \cdot \frac{\alpha_{0}}{\alpha}}\|f\|_{p}^{p-\frac{\alpha_{0}}{\alpha}}
\end{aligned}
$$

and if $p=\infty$, using (1.4) with $t=1$, we have

$$
\begin{aligned}
\|f\|_{\infty} & \leq C_{d} \max _{k \in \Omega}\left[\left(N^{d-1} a_{N, k}\right)\left|f\left(\xi_{N, k}\right)\right|\right] \\
& \leq C_{d}\left(\max _{k \in \Omega}\left(N^{d-1} a_{N, k}\right)^{\alpha}\left|f\left(\xi_{N, k}\right)\right|\right)^{\frac{1}{\alpha}}\|f\|_{\infty}^{1-\frac{1}{\alpha}} .
\end{aligned}
$$

Therefore, in either case, we have the desired inverse inequality

$$
\|f\|_{p} \leq C_{p, d, \alpha}\left(\frac{1}{N^{d-1}} \sum_{k \in \Omega}\left(N^{d-1} a_{N, k}\right)^{\alpha p}\left|f\left(\xi_{k}\right)\right|^{p}\right)^{\frac{1}{p}}, \quad \forall \alpha \geq 0
$$

with the usual change when $p=\infty$.

Proof of Theorem 1.2. We start with the proof of the equivalence (1.10). We first note that for each $j \geq 0, \sigma_{j}(f) \in \Pi_{2^{j}}$ and $\left\langle f, \psi_{j, k}\right\rangle=\sqrt{\lambda_{j, k}} \sigma_{j}(f)\left(x_{j, k}\right)$. Thus, by (1.6) and Lemma 5.2 with $\alpha=\frac{1}{2}$ it follows that for $j \geq 0$ and $0<p \leq \infty$,

$$
\left\|\sigma_{j}(f)\right\|_{p} \asymp 2^{-j(d-1)\left(\frac{1}{p}-\frac{1}{2}\right)}\left(\sum_{k \in \Lambda_{j}^{d}}\left|\left\langle f, \psi_{j, k}\right\rangle\right|^{p}\right)^{\frac{1}{p}},
$$

with the usual change when $p=\infty$. This together with Lemma 5.1 implies (1.10) for $1<p \leq \infty$. To show (1.10) for $0<p \leq 1$, by Lemma 5.1 it suffices to prove that for $f \in H^{p}$ and $j \geq 1$

$$
\left\|\sigma_{j} \circ \sigma_{j}(f)\right\|_{H^{p}} \leq C_{p, d, \phi}\left(\sum_{k \in \Lambda_{j}^{d}}\left|\left\langle f, \psi_{j, k}\right\rangle\right|^{p} 2^{j(d-1)\left(\frac{p}{2}-1\right)}\right)^{\frac{1}{p}} \leq C_{p, d, \phi}^{\prime}\left\|\sigma_{j}(f)\right\|_{H^{p}} .
$$

We note that

$$
\sigma_{j} \circ \sigma_{j}(f)(x)=\int_{\mathbb{S}^{d-1}} \sigma_{j}(f)(y) G_{j}(x \cdot y) d \sigma(y), \quad x \in \mathbb{S}^{d-1} .
$$

Hence, by the cubature formula (1.5) it follows that

$$
\sigma_{j} \circ \sigma_{j}(f)(x)=\sum_{k \in \Lambda_{j}^{d}} \lambda_{j, k} \sigma_{j}(f)\left(x_{j, k}\right) G_{j}\left(x \cdot x_{j, k}\right)=\sum_{k \in \Lambda_{j}^{d}}\left\langle f, \psi_{j, k}\right\rangle \psi_{j, k}(x),
$$


which together with Theorem 1.1 implies

$$
\left\|\sigma_{j} \circ \sigma_{j}(f)\right\|_{H^{p}} \leq C_{p, d, \phi}^{\prime}\left(\sum_{k \in \Lambda_{j}^{d}}\left|\left\langle f, \psi_{j, k}\right\rangle\right|^{p} 2^{j(d-1)\left(\frac{p}{2}-1\right)}\right)^{\frac{1}{p}},
$$

since $\sum_{k \in \Lambda_{j}^{d}} \chi_{B\left(x_{j, k}, 2^{-j} \pi\right)}(x) \leq C_{d}$. Thus, combining (5.3) with (5.1), taking into account the fact that $\left\|\sigma_{j}(f)\right\|_{p} \leq\left\|\sigma_{j}(f)\right\|_{H^{p}}$, we deduce (5.2).

It remains to prove the inequality (1.11). Without loss of generality, we may assume that only a finite number of the coefficients $a_{j, k}$ are nonzero. We then deduce from the definition and the Hardy-type inequality that

$$
|f|_{B_{\tau}^{\alpha}\left(H^{p}\right)} \leq C_{p, \tau}\left(\sum_{j=0}^{\infty} 2^{j \alpha \tau}\left\|\sum_{k \in \Lambda_{j}^{d}} a_{j, k} \psi_{j, k}\right\|_{H^{p}}^{\tau}\right)^{\frac{1}{\tau}},
$$

with the usual change when $\tau=\infty$. However, for $0<p<\infty$, by Theorem 1.1 we have

$$
\left\|\sum_{k \in \Lambda_{j}^{d}} a_{j, k} \psi_{j, k}\right\|_{H^{p}} \leq C_{p, d} 2^{j(d-1)\left(\frac{1}{2}-\frac{1}{p}\right)}\left(\sum_{k \in \Lambda_{j}^{d}}\left|a_{j, k}\right|^{p}\right)^{\frac{1}{p}},
$$

since $\sum_{k \in \Lambda_{j}^{d}} \chi_{B\left(x_{j, k}, 2^{-j} \pi\right)} \leq C_{d}$, while for $p=\infty$, we apply Lemma 2.2 to obtain

$$
\left|\psi_{j, k}(x)\right| \leq C_{d, \phi} 2^{\frac{j(d-1)}{2}} \min \left\{1,\left(2^{j} d\left(x, x_{j, k}\right)\right)^{-d}\right\},
$$

from which it follows that

$$
\begin{array}{r}
\left|\sum_{k \in \Lambda_{j}^{d}} a_{j, k} \psi_{j, k}(x)\right| \leq C_{d, \phi} 2^{j(d-1) / 2}\left(\max _{k \in \Lambda_{j}^{d}}\left|a_{j, k}\right|\right)\left|\sum_{k \in \Lambda_{j}^{d}} \min \left\{1,\left(2^{j} d\left(x, x_{j, k}\right)\right)^{-d}\right\}\right| \\
\leq C_{d, \phi} 2^{j(d-1) / 2}\left(\max _{k \in \Lambda_{j}^{d}}\left|a_{j, k}\right|\right)\left(\sum_{\substack{x_{j, k} \in B\left(x, \frac{\pi}{2 j}\right) \\
k \in \Lambda_{j}^{d}}} 1+\sum_{i=1}^{2^{j}-1} \sum_{\frac{i \pi}{2 j}<d\left(x, x_{j, k}\right) \leq \frac{(i+1) \pi}{2^{j}}} i^{-d}\right) \\
\leq C_{d, \phi} 2^{j(d-1) / 2}\left(\max _{k \in \Lambda_{j}^{d}}\left|a_{j, k}\right|\right)\left(1+\sum_{i=1}^{\infty} i^{-2}\right) \leq C_{d, \phi} 2^{j(d-1) / 2}\left(\max _{k \in \Lambda_{j}^{d}}\left|a_{j, k}\right|\right) .
\end{array}
$$

Now substituting (5.5) and (5.6) into (5.4) gives the desired inequality (1.11). This completes the proof.

\section{Proofs of Theorems 1.3 And 1.4}

Proof of Theorem 1.3. For a spherical cap $B=B\left(x_{j, k}, 2^{-j} \pi\right) \in \mathcal{B}$, we set $w_{B}(x)=$ $\left(1+2^{j} d\left(x, x_{j, k}\right)\right)^{-d-1}$. We define

$$
F(x):=\left(\sum_{B \in \mathcal{B}}\left|\left\langle f, \psi_{B}\right\rangle\right|^{\tau}|B|^{\left(-\frac{1}{2}-\frac{\alpha}{d-1}\right) \tau} w_{B}(x)\right)^{\frac{1}{\tau}} .
$$


Then by Theorem 1.2, $\|F\|_{\tau} \asymp|f|_{B_{\tau}^{\alpha}\left(H^{\tau}\right)}$. Thus, it will suffice to prove

$$
\left\|f-G_{n}^{p}(f)\right\|_{H^{p}} \leq C_{p, \alpha} n^{-\frac{\alpha}{d-1}}\|F\|_{\tau} .
$$

Recall that

$$
G_{n}^{p}(f)=\sum_{B \in \Gamma_{n}}\left\langle f, \psi_{B}\right\rangle \psi_{B},
$$

where $\Gamma_{n} \equiv \Gamma_{n, p, f}$ is a set of $n$ spherical caps $B \in \mathcal{B}$ such that

$$
\min _{B \in \Gamma_{n}}\left|\left\langle f, \psi_{B}\right\rangle\right||B|^{\frac{1}{p}-\frac{1}{2}} \geq \max _{B \in \mathcal{B} \backslash \Gamma_{n}}\left|\left\langle f, \psi_{B}\right\rangle\right||B|^{\frac{1}{p}-\frac{1}{2}} .
$$

We set

$$
\Sigma(x):=\left(\sum_{B \in \mathcal{B} \backslash \Gamma_{n}}\left|\left\langle f, \psi_{B}\right\rangle\right|^{q}\left|\psi_{B}(x)\right|^{q}\right)^{\frac{1}{q}},
$$

where and throughout the proof, $q=2$ if $p<\infty$, and $=1$ if $p=\infty$. It then follows by Theorem 1.1 that

$$
\left\|f-G_{n}^{p}(f)\right\|_{H^{p}} \leq C_{p}\|\Sigma\|_{p} .
$$

We claim that for $x \in \mathbb{S}^{d-1}$

$$
\Sigma(x) \leq C_{p, \alpha} n^{-\frac{\alpha}{d-1}}(F(x))^{\frac{\tau}{p}}\|F\|_{\tau}^{1-\frac{\tau}{p}},
$$

which combined with (6.2) will give the desired inequality (6.1).

To prove (6.3), we write, for $t>0$,

$$
\begin{aligned}
& \Sigma_{1}^{t}(x):=\left(\sum_{\substack{B \in \mathcal{B} \backslash \Gamma_{n} \\
|B| \geq t^{d-1}}}\left|\left\langle f, \psi_{B}\right\rangle\right|^{q}\left|\psi_{B}(x)\right|^{q}\right)^{\frac{1}{q}}, \\
& \Sigma_{2}^{t}(x):=\left(\sum_{\substack{B \in \mathcal{B} \backslash \Gamma_{n} \\
|B|<t^{d-1}}}\left|\left\langle f, \psi_{B}\right\rangle\right|^{q}\left|\psi_{B}(x)\right|^{q}\right)^{\frac{1}{q}} .
\end{aligned}
$$

We note that for $B \in \mathcal{B} \backslash \Gamma_{n}$,

$$
\left|\left\langle f, \psi_{B}\right\rangle\right||B|^{\frac{1}{p}-\frac{1}{2}} \leq n^{-\frac{1}{\tau}}\left(\sum_{\widetilde{B} \in \Gamma_{n}}\left|\left\langle f, \psi_{\widetilde{B}}\right\rangle\right|^{\tau}|\widetilde{B}|^{\frac{\tau}{p}-\frac{\tau}{2}}\right)^{\frac{1}{\tau}} \leq C_{p, \alpha} n^{-\frac{1}{\tau}}\|F\|_{\tau},
$$

and by Lemma 2.2 ,

$$
\left|\psi_{B}(x)\right| \leq C_{d}|B|^{-\frac{1}{2}}\left(w_{B}(x)\right)^{1+\frac{1}{\tau}} .
$$

Therefore, for $\Sigma_{1}^{t}(x)$, using (6.4) and (6.5), we have

$$
\begin{aligned}
\Sigma_{1}^{t}(x) & \leq C_{p, \alpha}\left(\sum_{B \in \mathcal{B} \backslash \Gamma_{n}|B| \geq t^{d-1}}|B|^{-\frac{q}{p}}\left(w_{B}(x)\right)^{\left(1+\frac{1}{\tau}\right) q}\right)^{\frac{1}{q}} n^{-\frac{1}{\tau}}\|F\|_{\tau} \\
& \leq C_{p, \alpha} n^{-\frac{1}{\tau}} t^{-\frac{d-1}{p}}\|F\|_{\tau},
\end{aligned}
$$


while for $\Sigma_{2}^{t}(x)$, using (6.5), we have

$$
\begin{aligned}
& \Sigma_{2}^{t}(x) \leq C_{p, \alpha}\left(\sum_{\substack{B \in \mathcal{B} \backslash \Gamma_{n} \\
|B| \leq t^{d-1}}}\left|\left\langle f, \psi_{B}\right\rangle\right|^{q}|B|^{-\frac{q}{2}-\frac{q \alpha}{d-1}}|B|^{\frac{q \alpha}{d-1}}\left(w_{B}(x)\right)^{\left(1+\frac{1}{\tau}\right) q}\right)^{\frac{1}{q}} \\
& \leq C_{p, \alpha} \max _{B \in \mathcal{B}}\left(\left|\left\langle f, \psi_{B}\right\rangle\right||B|^{-\frac{1}{2}-\frac{\alpha}{d-1}}\left(w_{B}(x)\right)^{\frac{1}{\tau}}\right)\left(\sum_{\substack{B \in \mathcal{B} \\
|B| \leq t^{d-1}}}|B|^{\frac{q \alpha}{d-1}}\left(w_{B}(x)\right)^{q}\right)^{\frac{1}{q}} \\
&(6.7) \quad \leq C_{p, \alpha} t^{\alpha}|F(x)| .
\end{aligned}
$$

Combining (6.6) with (6.7), we obtain

$$
\Sigma(x) \leq C_{p, \alpha}\left[n^{-\frac{1}{\tau}} t^{-\frac{d-1}{p}}\|F\|_{\tau}+t^{\alpha}|F(x)|\right],
$$

and the claim (6.3) then follows by taking $t=n^{-\frac{1}{d-1}}\left(\frac{\|F\|_{\tau}}{F(x)}\right)^{\frac{\tau}{d-1}}$. This completes the proof.

Proof of Theorem 1.4. First, we prove that for $f=\sum_{B \in \mathcal{B}} a_{B} \psi_{B}$ with only $n$ nonzero coefficients $a_{B}$,

$$
|f|_{B_{\tau}^{\alpha}\left(H^{\tau}\right)} \leq C_{p, \alpha} n^{\frac{\alpha}{d-1}}\|g\|_{p}
$$

where $g(x):=\max _{B \in \Lambda}\left(\left|a_{B}\right||B|^{-1 / 2} \chi_{B}(x)\right)$ and $\Lambda=\left\{B \in \mathcal{B}: a_{B} \neq 0\right\}$. Suppose $\Lambda=\left\{B_{1}, \ldots, B_{n}\right\}$ with $\left|B_{1}\right| \leq \ldots \leq\left|B_{n}\right|$. Set $E_{1}=B_{1}$ and $E_{j}=B_{j} \backslash \bigcup_{i=1}^{j-1} B_{i}$, $j \geq 2$. Then by Theorem 1.2, we have

$$
\begin{aligned}
|f|_{B_{\tau}^{\alpha}\left(H^{\tau}\right)}^{\tau} & \leq C_{p, \alpha} \int_{\mathbb{S}^{d-1}}\left(\sum_{j=1}^{n}\left|a_{B_{j}}\right|^{\tau}\left|B_{j}\right|^{-\frac{\tau}{2}-\frac{\alpha \tau}{d-1}} \chi_{B_{j}}(x)\right) d \sigma(x) \\
& =C_{p, \alpha} \sum_{i=1}^{n} \int_{E_{i}} \sum_{j=i}^{n}\left|a_{B_{j}}\right|^{\tau}\left|B_{j}\right|^{-\frac{\tau}{2}-\frac{\alpha \tau}{d-1}} \chi_{B_{j}}(x) d \sigma(x) \\
& \leq C_{p, \alpha} \sum_{i=1}^{n} \int_{E_{i}}\left(\sum_{\substack{B \in \mathcal{B} \\
|B| \geq\left|B_{i}\right|}}|B|^{-\frac{\alpha \tau}{d-1}} \chi_{B}(x)\right)|g(x)|^{\tau} d \sigma(x) \\
& \leq C_{p, \alpha} \sum_{i=1}^{n}\left|B_{i}\right|^{-\frac{\alpha \tau}{d-1}} \int_{E_{i}}|g(x)|^{\tau} d \sigma(x) \\
& \leq C_{p, \alpha} n^{1-\frac{\tau}{p}}\|g\|_{p}^{\tau}=C_{p, \alpha} n^{\frac{\alpha \tau}{d-1}}\|g\|_{p}^{\tau},
\end{aligned}
$$

which gives (6.8).

Next, we show

$$
\left\|G_{n}^{p}(f)\right\|_{B_{\tau}^{\alpha}\left(H^{\tau}\right)} \leq C_{p, \alpha} n^{\frac{\alpha}{d-1}}\|f\|_{H^{p}}
$$

By (6.8), it suffices to prove that

$$
\left\|\max _{B \in \Gamma_{n}}\left(\left|\left\langle f, \psi_{B}\right\rangle\right||B|^{-1 / 2} \chi_{B}(x)\right)\right\|_{p} \leq C_{p, \alpha}\|f\|_{H^{p}} .
$$


(6.9) for $0<p<\infty$ follows directly from Theorem 1.1, while for $p=\infty$ follows by the following inequality, which can be easily deduced by Lemma 2.2:

$$
\max _{B \in \mathcal{B}}\left|\left\langle f, \psi_{B}\right\rangle\right||B|^{-\frac{1}{2}} \chi_{B}(x) \leq C_{d, \phi} M(f)(x), \quad x \in \mathbb{S}^{d-1} .
$$

This completes the proof.

\section{ACKNowledgements}

The author would like to express his sincere gratitude to an anonymous referee for many helpful comments on this paper.

\section{REFERENCES}

[ADS] A. Askari-Hemmat, M. A. Dehghan, and M. Skopina, Polynomial wavelet-type expansions on the sphere, Math. Notes 74 (2003), no. 2, 292-300. MR2023771|(2005a:42024)

[AV] J.P. Antoine and P. Vandergheynst, Wavelets on the $n$-sphere and related manifolds, J. Math. Phys. 39 (1998), 3987-4008. MR.1633175 (99e:42045)

[BC] A. Bonami and J. L. Clerc, Sommes de Cesàro et multiplicateurs des dèveloppments en harmonique sphériques, Trans. Amer. Math. Soc. 183 (1973), 223-263. MR0338697 $(49: 3461)$

[BD] G. Brown and F. Dai, Approximation of smooth functions on compact two-point homogeneous spaces, J. Funct. Anal. 220 (2005), no. 2, 401-423. MR2119285 (2005m:41054)

[C] L. Colzani, Hardy spaces on unit spheres, Boll. Un. Mat. Ital. C(6) 4 (1985), no. 1, 219-244. MR0805216 (87d:42031)

[CTW] L. Colzani, M. H. Taibleson and G. Weiss, Maximal estimates for Cesàro and Riesz means on spheres, Indiana Univ. Math. J. 33 (1984), no. 6, 873-889. MR0763947 (86g:43012)

[DDSW] S. Dahlke, W. Dahmen, E. Schmidt and I. Weinrich, Multiresolution analysis on $\mathbb{S}^{2}$ and $\mathbb{S}^{3}$, Numer. Funct. Anal. Optim. 16 (1995), 19-41. MR1322896 (96a:42044)

[DJP] R. A. DeVore, B. Jawerth and V. Popov, Compression of wavelet decompositions, Amer. J. Math. 114 (1992), no. 4, 737-785. MR1175690(94a:42045)

[DP] R. A. DeVore, V. Popov, Interpolation spaces and nonlinear approximation, Lecture Notes in Mathematics, Vol. 1302, 191-205, Springer-Verlag, New York, 1988. MR0942269 (89d:41035)

[DPY] R. A. DeVore, P. Petrushev and X. M. Yu, Nonlinear wavelet approximation in the space $C\left(R^{d}\right)$, In: Progress in approximation theory (A.A. Gonchar, E. B. Saff, eds.), 261-283. New York: Springer-Verlag, 1992. MR 1240786 (94h:41070)

[FGS] W. Freeden, T. Gervens and M. Schreiner, Constructive Approximation on the Sphere: with Applications to Geomathematics, Clarendon Press, Oxford, 1998. MR1694466 (2000e:41001)

[FJ] M. Frazier and B. Jawerth, A discrete transform and decompositions of distribution spaces, J. Funct. Anal. 93 (1990), no. 1, 34-170. MR1070037 (92a:46042)

[G] J. Göttelmann, Locally supported wavelets on manifolds with applications to the $2 D$ sphere, Appl. Comput. Harmon. Anal. 7 (1999), 1-33. MR1699606 (2000j:42051)

[GJ] S. Grellier and P. Jaming, Harmonic functions on the real hyperbolic ball. II. Hardy-Sobolev and Lipschitz spaces, Math. Nachr. 268 (2004), 50-73. MR2054532 (2005a:46067)

[HW] E. Hernández and G. Weiss, A first course on wavelets, CRC Press, Boca Raton, FL, 1996. MR.1408902 (97i:42015)

[Jia] Rong-Qing Jia, A Bernstein-type inequality associated with wavelet decomposition, Constr. Approx. 9 (1993), 299-318. MR1215774 (94h:41026)

[KL] K. Kolarov and W. Lynch, Compression of functions defined on surfaces of $3 D$ objects, Data Compression Conference (DCC 97), March 25-27 (1997), 281-290.

[L] P. G. Lemarié-Rieusset, Base dóndelettes sur les groupes de Lie stratifiés (French), Bull. Soc. Math. France 117 (1989), no. 2, 211-232. MR1015808 (90j:42066) 
[MP] H. N. Mhaskar and J. Prestin, Polynomial frames: a fast tour, Approximation theory XI: Gatlinburg 2004, 287-318, Mod. Methods Math., Nashboro Press, Brentwood, TN, 2005. MR 2126687 (2005k:42087)

[MNW] H.N. Mhaskar, F.J. Narcowich and J.D. Ward, Spherical Marcinkiewicz-Zygmund inequalities and positive quadrature, Math. Comp. 70 (2001), 1113-1130 (Corrigendum: Math. Comp. 71 (2001) 453-454). MR.1710640 (2002a:41032)

[MNPW] H. N. Mhaskar, F. J. Narcowich, J. Prestin and J. D. Ward, Polynomial frames on the sphere, Adv. Comput. Math. 13 (2000), no. 4, 387-403. MR1826335(2002a:42021)

[NW] F. J. Narcowich, J. D. Ward, Nonstationary wavelets on the m-sphere for scattered data, Appl. Comput. Harmon. Anal. 3 (1996), no. 4, 324-336. MR1420501 (97h:42020)

[NPW] F. J. Narcowich, P. Petrushev and J. D. Ward, Localized tight frames on spheres, preprint.

[R] Kh. P. Rustamov, On the approximation of functions on the sphere, Izv. Akad. Nauk SSSR Ser. Mat. 59 (1993), 127-148. MR1252759 (94k:41051)

[SS1] P. Schröder and W. Sweldens, Spherical wavelets: Efficiently representing functions on a sphere, Computer Graphics (SIGGRAPH 95 Proceedings) (1995), 161-172.

[SS2] P. Schröder and W. Sweldens, Spherical Wavelets: Texture Processing, In P. Hanrahan and W. Purgathofer, editors, Rendering Techniques 95, pp. 252-263, Springer-Verlag, Wien, New York, 1995.

[SW] E. M. Stein and G. Weiss, Introduction to Fourier analysis on Euclidean spaces, Princeton Univ. Press, Princeton, NJ, 1971. MR0304972 (46:4102)

[S] R. Strichartz, Multipliers for spherical harmonic expansions, Trans. Amer. Math. Soc. 167 (1972), 115-124. MR0306823 (46:5945)

[Sz] G. Szegö, Orthogonal polynomials, American Mathematical Society Colloquium Publications 23, Revised ed., American Mathematical Society, Providence, RI, 1959. MR0106295 (21:5029)

[T1] V. N. Temlyakov, The best m-term approximation and greedy algorithms, Adv. Comput. Math. 8 (1998), no. 3, 249-265. MR.1628182 (99f:41037)

[T2] V. N. Temlyakov, Nonlinear methods of approximation, Found. Comput. Math. 3 (2003), no. 1, 33-107. MR 1951502 (2003j:41029)

Department of Mathematical and Statistical Sciences, CAB 632, University of AlBerta, Edmonton, Alberta, Canada T6G 2G1

E-mail address: dfeng@math.ualberta.ca 